\title{
Competition under Time-Varying Demands and Dynamic Lot Sizing Costs
}

\author{
Awi Federgruen \\ Columbia University \\ Graduate School of Business
}

\author{
Joern Meissner \\ Lancaster University \\ Management School
}

Submitted June 2004, Revision May 2008

\begin{abstract}
We develop a competitive pricing model which combines the complexity of time-varying demand and cost functions and that of scale economies arising from dynamic lot sizing costs. Each firm can replenish inventory in each of the $T$ periods into which the planning horizon is partitioned. Fixed as well as variable procurement costs are incurred for each procurement order, along with inventory carrying costs. Each firm adopts, at the beginning of the planning horizon, a (single) price to be employed throughout the horizon. Based on each period's system of demand equations, these prices determine a time series of demands for each firm, which needs to service them with an optimal corresponding dynamic lot sizing plan. We establish the existence of a price equilibrium and associated optimal dynamic lotsizing plans, under mild conditions. We also design efficient procedures to compute the equilibrium prices and dynamic lotsizing plans.
\end{abstract}




\section{Introduction}

Determining the 'right' price to charge for a product is a complex task. A voluminous literature in economics and marketing has been devoted to models which prescribe how prices should be set in industries in which a limited number of competing firms offer similar products, which may therefore be viewed as substitutes. These papers typically model the interaction among competitors as a noncooperative game, see Vives (2000) and Tirole (1988) for survey texts.

More recently, operations management papers have demonstrated that the operational environment and associated cost structures may have a fundamental impact on the equilibrium behavior in the industry, in general, and the resulting price levels in particular. See CACHON (2003) for a recent survey. Little remains known, however, about how prices should be set in a competitive environment, in the simultaneous presence of two other major complications:

(i) time dependent demand functions and cost parameters, and

(ii) scale economies in the operational costs.

In contrast, progress has been made in addressing either one of these factors by itself. For example, Bernstein and Federgruen (2003) address a setting where each firm incurs fixed as well as variable procurement costs along with (linear) inventory carrying costs. However, the model assumes an infinite horizon setting with time-invariant demand functions and cost parameters. Here the long-run average operational costs are given by the simple closed-form Economic Order Quantity (EOQ) cost function, i.e. the costs are given by the sum of a term that is proportional to the demand value itself and one that is proportional with the square root of the demand value, thus reflecting scale economies. CACHON and HARKER (2002) similarly consider, for an industry with two firms, a setting with a single set of time-invariant demand functions and with a closed form cost function given by a concave power function of the demand volume, (possibly in conjunction with a linear cost component), once again to reflect scale economies. Other than the EOQ-cost model above, the authors show that their cost structure arises in a specific service competition model. The only oligopoly models with stochastic demands avoid both complications (i) and (ii), see e.g. Bernstein and Federgruen (2004).

A stream of marketing papers addresses competitive pricing problems under time-dependent demand functions, however with simple linear cost functions, and under the assumption that each period's demand is procured in the same period, i.e. no inventories are carried. These papers 
include Kalish (1983), Eliashberg and Jeuland (1986), Clarke and Dolan (1984), Rao and Bass (1985), and Dockner and Jorgensen (1988). See Elmaghraby and Keskinocak (2003) for a survey. Perakis and Sood (2003, 2004) and Kachani, Perakis and Simon (2004) also address competitive pricing problems under time-varying demand functions. Since each firm starts the planning horizon with a known inventory and inventories cannot be replenished at any time during the horizon, these models consider no replenishment costs.

This paper appears to develop the first competitive pricing model which combines the complexity of time-varying demand and cost functions and that of scale economies arising from dynamic lot sizing costs. Each firm can replenish inventory in each of the $T$ periods into which the planning horizon is partitioned. Fixed as well as variable procurement costs are incurred for each procurement order, along with inventory carrying costs. Each firm adopts, at the beginning of the planning horizon, a (single) price to be employed throughout the horizon. Based on each period's system of demand equations, these prices determine a time series of demands for each firm, which needs to service them with an optimal corresponding dynamic lot sizing plan. Scale economies always create major analytical complications in the study of price equilibria, see e.g. VIVES (2000) and CACHON and Harker (2002). In our case, the problems are compounded by the fact that the cost structure can not be represented as a closed form analytical function of the (time series of) demand volume(s). We model each firm's time dependent demand function as a time invariant function multiplied by a firm- and period-dependent 'seasonality' factor. In $\S 6$, we generalize our results to allow for additive seasonality terms.

We establish, under mild conditions, the existence of a price equilibrium and associated optimal dynamic lotsizing plans. We also design efficient procedures to compute the equilibrium prices and dynamic lotsizing plans. Finally, we characterize how the equilibrium is affected by changes in various cost and demand function parameters. Much of the analysis focuses on an individual firm's best response problem, i.e. the characterization of the optimal price and lot sizing strategy, in response to a given set of prices adopted by the firm's competitors. This best response problem is of interest in its own right. For the case of constant, but firm dependent, setup costs, we design an efficient algorithm whose computational effort involves $O\left(T^{2}\right)$ elementary operations and $O(T)$ maximizations of a single variable concave function. (For many classes of demand functions, these maximizations can be performed in closed form). For the generalized model in $\S 6$, with additive 
seasonality terms and time-dependent setup costs, the recent $O\left(T^{3} \log T\right)$ procedure by VAN DEN Heuvel and Wagelsmans (2004) can be used.) The algorithm for the best response problem is used repeatedly when computing the overall price and lotsizing equilibrium in the industry.

We also conduct a numerical study, which, among others, reveals the following insights: contrary to folklore, it is not always best for a firm to operate under time-invariant demand functions or cost parameters. Even in equilibrium, all firms in the industry may be better off under certain types of seasonality patterns in the demand or cost parameters. When the above mild existence conditions for a (unique) equilibrium fail to hold, the industry may switch from having a unique equilibrium to either having none or multiple equilibria, depending on which seasonality pattern prevails.

One of the most fundamental assumptions in our model is that each firm maintains a constant price throughout the season. In many industries this is the practice, either because mid-season price changes can not be implemented (e.g. catalog sales) or because they are managerially undesirable. For example, in many retail operations it is considered unacceptable to raise prices during the season and at most one or two markdowns in a single season are as much as contemplated. Empirical analysis has documented that prices for certain goods are extremely 'sticky', i.e. they change very slowly over time, if at all. CARLTON (1986), for example, has investigated price data for industrial buyers over a 10 year period and has concluded that the price rigidity in many industries is striking. KASHYAP (1995) analyzed the data from catalog sales and observed that the prices typically remain constant over several seasons, beyond the total of a year. As a third example, CECCHETTI (1986) has studied newsstand prices for some 40 American magazines over a period of close to 20 years. This author concluded that prices exhibited remarkable rigidity to the extent of dropping in real (i.e. inflation corrected) value by as much as a quarter before a price adjustment is implemented. Finally, BLINDER ET AL (1998) document and explain the multitude of reasons why companies maintain 'sticky' prices. (As demonstrated below, at least the best response problems are considerably easier under the alternative assumption where prices may be varied in each period.)

Beyond the competition models mentioned above, we now give a brief literature review. Even within the context of optimization models with a single decision maker, the integration of pricing and inventory strategies has only recently received the attention it deserves, even though the seminal papers on dynamic lot sizing by Wagner and Whitin (1958, 1959) and WAgner (1960), 45 years ago, already addressed the need to integrate pricing and production planning decisions. We 
refer to Eliashberg and Steinberg (1993) for a survey of early work and to Elmaghraby and KESKINOCAK (2003) for a more recent survey.

Thomas (1970) addressed the dynamic lot sizing problem in which each period's demand depends (exclusively) on the price charged during this period according to a period-specific demand function. Assuming prices can be changed arbitrarily from one period to the next and that demand in a given period is independent of the prices offered in other periods, the author shows that an optimal plan can be found by a simple extension of the classical shortest path method by WAGNER and Whitin (1958): As in the classical dynamic lot sizing problem with exogeneously specified demands, it is easily verified that replenishment orders should only be placed in periods with zero starting inventory. In view of this observation, the optimal procurement plan can thus again be described as the shortest path in a network in which each period is represented as a node and traversing an arc from period $i$ to period $j$ corresponds to the decision to satisfy all demands in period $i, i+1, \ldots, j-1$ from a single order delivered in period $i$. The only difference with the classical WAGNER-WHITIN shortest path procedure is that evaluation of the (optimal) cost or profit value of any arc $(i, j)$ now involves optimizing a closed form expression over the prices in periods $i, i+1, \ldots, j-1$. Thus, dynamic lot sizing problems with arbitrary time-dependent prices are radically simpler than under the requirement that a single constant price be used.

KunReuther and Schrage (1973) were the first to address the best response problem, indeed for the generalized model in $\S 6$. These authors propose a heuristic procedure which also generates an upper and lower bound for the optimal price. VAN DEN HEuvel and Wagelsmans (2004) developed an $O\left(T^{3} \log T\right)$ algorithm, based on KunReuther and Schrage's heuristic. Gilbert (1999) considers the special case of the best response problem in which all cost parameters remain constant over the entire planning horizon. Our procedure for the best response problem is based on GILBERT's approach but reduces the computational complexity by an order of magnitude $\left(O\left(T^{2}\right)\right.$ compared to $O\left(T^{3}\right)$ complexity), while addressing more general parameter settings. This complexity reduction is particularly important in our competition model where best response problems need to be solved repeatedly and for each of the $N$ firms in the industry.

The remainder of the paper is organized as follows: In $\S 2$ we specify the competition model and the notation. $\S 3$ is devoted to the best response problem. The equilibrium analysis for the competitive model is carried out in $\S 4$. $\S 5$ reports on a numerical study, while $\S 6$ discusses conclusions 
and various generalizations of the model.

\section{Model and Notation}

We consider an industry with $N$ firms, each selling a distinct item or product brand. We refer to the item sold by firm $i$ as item $i=1, \ldots, N$. The different items are (close) substitutes of each other, e.g. different brands of 19" television sets, digital cameras, notebook computers, sport utility vehicles, tooth paste etc. We consider a planning horizon of $T$ periods. If the firms face a natural sales season introducing a new model or variant in each season, a natural choice of $T$ arises, e.g. $T=52$ weeks in the automobile manufacturing industry operating with a weekly production and sales schedule.

Each firm selects a (single) price to be used throughout the season. (See $\S 1$ for a discussion of this assumption.) Each firm's demand in each period depends potentially on the complete vector of prices selected in the industry according to a general time-dependent demand function. Let

$$
\begin{aligned}
p^{i} & =\text { the price chosen by firm } i=1, \ldots, N \text { from an interval }\left[p^{i, \text { min }}, p^{i, \max }\right] \\
d_{t}^{i} & =d_{t}^{i}\left(p^{1}, \ldots, p^{N}\right)=\text { the demand faced by firm } i \text { in period } t=1, \ldots, T
\end{aligned}
$$

Multiplicative seasonality factors characterize the demand functions' time dependence:

$$
d_{t}^{i}(p)=\beta_{t}^{i} \delta^{i}(p) \quad \forall \quad i=1, \ldots, N ; t=1, \ldots, T
$$

with $\delta^{i}(p)$ the deseasonalized demand function for firm $i, i=1, \ldots, N$. Depending on the period's seasonality factor, a firm's demand is, in a given period, scaled up $\left(\beta_{t}^{i}>1\right)$ or down $\left(\beta_{t}^{i}<1\right)$ compared to the deseasonalized norm. Without loss of generality, we normalize the seasonality factors such that:

$$
\frac{1}{T} \sum_{t=1}^{T} \beta_{t}^{i}=1, \quad i=1, \ldots, N
$$

(In $\S 6$, we discuss generalizations of (1), with additive seasonality terms.)

The deseasonalized functions $\delta^{i}(p)$ are continuously differentiable, with $\frac{\partial \delta^{i}(p)}{\partial p^{i}}<0$, i.e. a price increase results in a decrease of the demand volume. Since the demand function $\delta^{i}(p)$ is strictly 
decreasing in $p^{i}$, it is possible to derive an inverse demand function $p^{i}=\phi^{i}\left(\delta^{i} \mid p^{-i}\right)$. We only assume that firm i's revenue function $R^{i}\left(\delta^{i} \mid p^{-i}\right)=\delta^{i} \phi\left(\delta^{i} \mid p^{-i}\right)$ is concave in the demand volume $\delta^{i}$. Linear functions constitute an important special case:

$$
\delta^{i}(p)=a^{i}-b^{i} p_{i}+\sum_{j \neq i} \theta_{j}^{i} p^{j} \quad i=1, \ldots, N
$$

Without loss of generality, we assume that the Dominant Diagonal condition applies:

$$
b^{i}>\sum_{j \neq i} \theta_{j}^{i} \quad, \quad i=1, \ldots, N
$$

It merely precludes completely unrealistic situations where an across the board price increase in the industry results in an increase of a firm's sales volume. The Cobb-Douglas functions represent another important class of demand functions:

$$
\delta^{i}(p)=a^{i}\left(p^{i}\right)^{-b^{i}} \prod_{j \neq i}\left(p^{j}\right)^{\theta_{j}^{i}}, \quad \text { with } \quad a^{i}, \theta_{j}^{i}>0 \quad \text { and } \quad b^{i}>1
$$

$b^{i}$ is the absolute elasticity of firm i's demand with respect to its own price, while $\theta_{j}^{i}$ denotes the cross elasticity with respect to competitor $j$ 's price. Under the Cobb-Douglas structure, these price elasticities are therefore constant, i.e. independent of the price levels.

The firms procure their goods by a production and distribution process which, in principle, allows for inventory replenishments at the beginning of each period. As in standard dynamic lot sizing problems, we assume that fixed as well as variable procurement costs are incurred as well as inventory carrying costs which are proportional to each end-of-the-period inventory. All cost parameters may fluctuate over the course of the planning horizon in arbitrary ways. Thus, for all $i=1, \ldots, N$ and $t=1, \ldots, T$ let

$K_{t}^{i}=$ the fixed setup cost for a procurement batch delivered to firm $i$ in period $t$,

$c_{t}^{i}=$ the procurement cost rate for a procurement batch delivered to firm $i$ in period $t$,

$h_{t}^{i}=$ the cost of carrying a unit of item $i$ in inventory at the end of period $t$.

Each firm $i$ thus selects a price $p^{i}$ as well as a complete procurement schedule for the entire planning horizon to support the demand stream $\left\{d_{t}^{i}(p)\right\}$ which arises from the collective price 
choices. Note that his price $p^{i}$ affects the profits earned by all firms in the industry via its impact on each firm's demand function and hence each firm's demand stream. At the same time, the procurement schedule selected by firm $i$ impacts only his own profit measure. It is thus possible to conceptualize the competition model as a Bertrand price competition game between $N$ firms, in which each firm makes a single competitive choice, i.e. its price level. (Alternatively, one may assume that each firm i selects a basic deseasonalized target volume $\delta^{i}$. This results in a vector of prices $\mathrm{p}$ which satisfy the demand equations $\delta^{i}=\delta^{i}(p), i=1, \ldots, N$. We discuss this Cournot competition variant at the end of $\S 6$ ). The game is characterized by the profit functions:

$$
\begin{aligned}
\pi^{i}(p)= & \text { the profit earned by firm } i \text { under the price-vector } p \text {, assuming firm } i \text { adopts an } \\
& \text { optimal dynamic lot sizing schedule given the demand stream }\left\{d_{t}^{i}(p): t=1, \ldots, T\right\} \\
= & p^{i} \sum_{t=1}^{T} d_{t}^{i}(p)-C^{i}(p) \quad i=1, \ldots, N, \quad \text { where }
\end{aligned}
$$

$C^{i}(p)=$ the minimum total operating costs for firm $i$ to service the demand stream $\left\{d_{t}^{i}(p)\right\}$.

The difficulty in analyzing the competition model and in characterizing its equilibrium behavior, stems from the complexity of the cost functions $C^{i}(p)$. Clearly, the function $C^{i}(p)$ cannot be represented as an analytical closed form expression. The function can be evaluated for any given price vector $p$ in $O\left(T^{2}\right)$ time using the standard Wagner-Whitin shortest path procedure and in $O(T \log T)$ time by one of the methods in Aggarwal and Park (1993), Federgruen and Tzur (1991), or Van Hoesel and Wagelmans (1992). Clearly $C^{i}(p)$ depends on the price vector $p$ 'only' through the demand sequence $\left\{d_{t}^{i}(p)=d_{t}^{i}\right\}$.

Lemma $1 C^{i}$ is a piecewise linear concave function of the demand sequence $\left\{d_{t}^{i}=d_{t}^{i}(p)\right\}$.

Proof: Fix a price vector $p \in R^{N}$. Assume firm $i$ chooses to replenish its inventory in the set of periods $\Theta=\left\{t_{1}=1, t_{2}, \ldots, t_{n}\right\}$. It is well known that a Zero-Inventory Ordering (ZIO) policy is optimal, so the optimal cost under this sequence of order periods is given by:

$$
\begin{aligned}
C^{i}(p \mid \Theta)= & \sum_{l=1}^{n} K_{t_{l}}^{i}+\sum_{l=1}^{n} c_{t_{l}}^{i} \sum_{r=t_{l}}^{t_{l+1}-1} d_{r}^{i}(p) \\
& +\sum_{l=1}^{n} \sum_{r=t_{l}}^{t_{l+1}-1} h_{r}^{i}\left(d_{r+1}^{i}(p)+\cdots d_{t_{l+1}-1}^{i}(p)\right)
\end{aligned}
$$


where $t_{n+1}=T+1$. Finally, $C^{i}(p)=\min \left\{C^{i}(p \mid \Theta) \mid \Theta \in 2^{\{1, \ldots, T\}}\right\}$, i.e. $C^{i}(p)$ is the minimum of $\left(2^{T}-1\right)$ linear functions and is therefore concave in the vector $\left\{d_{t}^{i}: t=1, \ldots, T\right\}$.

As a corollary of the above Lemma we obtain that, if all demand functions are linear, the cost functions $C^{i}(p)$ are piecewise linear and jointly concave in the price vector $p$, itself. Unfortunately, this characterization is by itself insufficient to conduct the equilibrium analyses. First, concave cost functions reflecting economies of scale create major analytical difficulties for equilibrium analyses, see Vives (2000) and Cachon and Harker (2002). Second, the fact that $C^{i}(p)$ is not available in closed form generates additional complexities.

Before providing a complete characterization of the industry's equilibrium behavior, we first analyze an individual firm's best response problem. Thus, for any $i=1, \ldots, N$, let

$\pi^{i}\left(p^{i} \mid p^{-i}\right)=$ the profit for firm i when choosing price $p^{i}$, given his competitors' prices $p^{-i}=$ $\left(p^{1}, \ldots, p^{i-1}, p^{i+1}, \ldots, p^{N}\right)$ and assuming firm i minimizes operating costs to service the resulting demand stream $\left\{d_{t}^{i}(p)\right\}$.

Firm i's best response problem, given his competitors' prices $p^{-i}$, can then be formulated as:

$$
\pi^{* i}\left(p^{-i}\right)=\max _{p^{i}} \pi^{i}\left(p^{i} \mid p^{-i}\right)
$$

\section{The best response problem under constant setup costs}

In this $\S$, we analyze a given firm i's best response problem (8) in the important special case where

its setup cost remains constant across time $\left(K_{i}^{i}=\cdots=K_{T}^{i} \stackrel{\text { def }}{=} K^{i}\right)$, so that, for any sequence of order periods $\Theta=\left\{t, \ldots, t_{n}\right\}$ with $n$ setup periods (see $(7)$ ):

$$
\begin{aligned}
& C^{i}(p \mid \Theta)=n K^{i}+\delta^{i}(p)\left\{\sum_{l=1}^{n} c_{t_{l}}^{i} \sum_{r=t_{l}}^{t_{l+1}-1} \beta_{r}^{i}+\sum_{l=1}^{n} \sum_{r=t_{l}}^{t_{l+1}-1} h_{r}^{i}\left(\beta_{r+1}^{i}+\cdots+\beta_{t_{l+1}-1}^{i}\right)\right\} \quad \text { and } \\
& C^{i}(p)=\min _{\Theta \in 2^{\{1, \ldots, T\}}} C^{i}(p \mid \Theta)=\min _{n} \min _{\Theta \mid n} C^{i}(p \mid \Theta)=\min _{n}\left\{n K^{i}+\delta^{i}(p) F_{n}^{i}(T)\right\}
\end{aligned}
$$


where

$$
\begin{aligned}
F_{n}^{i}(t)= & \text { minimum total variable procurement and holding costs in periods }\{1, \ldots, t\} \text { for }(11) \\
& \text { firm i, when exactly } \mathrm{n} \text { orders are placed in the first } \mathrm{t} \text { periods, assuming the } \\
& \text { firm's demand stream is given by the seasonality factors }\left\{\beta_{1}^{i}, \ldots, \beta_{t}^{i}\right\} \text { and } \\
& \text { assuming only ZIO policies are considered; } t=1, \ldots, T ; \quad n=1, \ldots, t, i=1, \ldots, N .
\end{aligned}
$$

Since the overall optimal replenishment strategy for firm $i$, under any price vector $p$, employs Zero Inventory Ordering (ZIO), we may, in the definition of $F_{n}^{i}(t)$, confine ourselves to such strategies as well. Also, since $F_{n}^{i}(t)$ is the minimum cost in a dynamic lot sizing problem with zero fixed order costs, we may, in the definition of $F_{n}^{i}(t)$ replace the requirement that exactly $n$ orders be placed, by the (relaxed) requirement that at most $n$ orders be placed.

The representation of the minimum total cost as the minimum of $T$ affine functions of $\delta^{i}(p)$ was first obtained by GILBERT (1999), for the special case where all cost parameters remain constant. Gilbert proceeds to compute the numbers $\left\{F_{n}^{i}(T): n=1, \ldots, T\right\}$ with a straightforward $O\left(T^{3}\right)$ recursion.

In the next subsection we develop an $O\left(T^{2}\right)$ procedure to compute the matrix of values $\left\{F_{n}^{i}(t) ; t=1, \ldots, T ; n=1, \ldots, T\right\}$. Thus, assuming these values have been determined, it follows from (3) that the best response problem (8) reduces to:

$$
\begin{aligned}
\pi^{* i}\left(p^{-i}\right) & =\max _{p^{i}} \max _{n}\left\{T p^{i} \delta^{i}(p)-n K^{i}-\delta^{i}(p) F_{n}^{i}(T)\right\} \\
& =\max _{n} \max _{p^{i}}\left\{\left[T p^{i}-F_{n}^{i}(T)\right] \delta^{i}\left(p^{i} \mid p^{-i}\right)-n K^{i}\right\} \\
& =\max _{n} \max _{\delta^{i}}\left\{T R^{i}\left(\delta^{i} \mid p^{-i}\right)-F_{n}^{i}(T) \delta^{i}-n K^{i}\right\}
\end{aligned}
$$

In other words, the best response problem reduces to $\mathrm{T}$ maximization problems of a concave function of a single variable $\delta^{i}$. If the revenue function $R^{i}\left(\delta^{i} \mid p^{-i}\right)$ is strictly concave, its derivative $R^{i^{\prime}}\left(\cdot \mid p^{-i}\right)$ is strictly decreasing and has an inverse function $\left(R^{i^{\prime}}\right)^{-1}\left(\cdot \mid p^{-i}\right)$. The maximizing value of $\delta^{i}$ in (12) is then given by $\delta^{* i}(n)=\left(R^{i^{\prime}}\right)^{-1}\left(\frac{F_{n}^{i}(T)}{T} \mid p^{-i}\right)$. Substituting this demand value into (12), we conclude that the solution of the best response problem reduces to the determination of $T$ closed form expressions (for $n=1, \ldots, T$ ) involving the inverse derivative revenue function 
$\left(R^{i^{\prime}}\right)^{-1}\left(\cdot \mid p^{-i}\right)$. Assuming this function can be evaluated in constant time, the best response problem can thus be solved in $O\left(T^{2}\right)$ time.

Example 1: Assume the deseasonalized demand functions $\delta^{i}(p)$ are linear, as in (3). This implies that $p^{i}=\left[a^{i}+\sum_{j \neq i} \theta_{j}^{i} p^{j}-\delta^{i}\right] / b^{i}$, giving rise to a quadratic revenue function $R^{i}\left(\cdot \mid p^{-i}\right)$ and an optimal deseasonalized demand volume, given $n$ orders, of

$$
\delta^{* i}(n)=\left[\left(\frac{a^{i}+\sum_{j \neq i} \theta_{j}^{i} p^{j}}{2}\right)-\frac{b^{i} F_{n}^{i}(T)}{2 T}\right]^{+}
$$

and a corresponding price

$$
p^{* i}=\left[a^{i}+\sum_{j \neq i} \theta_{j}^{i} p^{j}-\delta^{* i}\right] / b^{i}
$$

Substituting this value into (12), we obtain:

$$
\pi^{* i}\left(p^{-i}\right)=\max _{n=1, \ldots, T}\left\{\begin{aligned}
{\left[\frac{\sqrt{T}}{2 \sqrt{b^{i}}}\left(a^{i}+\sum_{j \neq i} \theta_{j}^{i} p^{j}\right)-\frac{\sqrt{b^{i}}}{2 \sqrt{T}} F_{n}^{i}(T)\right]^{2}-n K^{i} } & , \text { if } \quad \delta^{* i}(n)>0 \\
-n K^{i} & , \text { otherwise, }
\end{aligned}\right.
$$

a closed form expression in terms of the values $\left\{F_{1}^{i}(T), F_{2}^{i}(T), \ldots, F_{T}^{i}(T)\right\}$.

Example 2: Assume the demand functions are of the Cobb-Douglas type in (5). Thus,

$$
p^{i}=\left(\delta^{i}\right)^{-\frac{1}{b^{i}}} a^{i \frac{1}{b^{i}}} \prod_{j \neq i}\left(p^{j}\right)^{\frac{\theta_{j}^{i}}{b^{i}}}
$$

so that $\delta^{* i}(n)$,the optimal deseasonalized demand volume, given a total of $n$ orders during he planning horizon, is the value of $\delta^{i}$ which maximizes the concave function $T\left(\delta^{i}\right)^{1-\frac{1}{b^{i}}} a^{i \frac{1}{b^{i}}} \prod_{j \neq i}\left(p^{j}\right)^{\frac{\theta_{j}^{i}}{b^{i}}}-$ $F_{n}^{i}(T) \delta^{i}$. Thus,

$$
\delta^{* i}(n)=T^{b^{i}}\left(1-\frac{1}{b^{i}}\right)^{b^{i}} a^{i} \prod_{j \neq i}\left(p^{j}\right)^{\theta_{j}^{i}}\left[F_{n}^{i}(T)\right]^{-b^{i}}
$$


and after some algebra,

$$
\pi^{* i}\left(p^{-i}\right)=\max _{n=1, \ldots, T}\left\{\frac{a^{i} T^{b^{i}}}{b^{i}}\left(1-\frac{1}{b^{i}}\right)^{b^{i}-1} \prod_{j \neq i}\left(p^{j}\right)^{\theta_{j}^{i}}\left[F_{n}^{i}(T)\right]^{-b^{i}+1}-n K^{i}\right\}
$$

The multiplicative form of the expression for $\delta^{* i}(n)$ shows that, given any number of orders $n$, the percentage change in the optimal deseasonalized demand value or the optimal price, due to any change in the seasonality pattern, holding and variable order cost rates, is independent of what prices are offered by the competing firms. The smaller the absolute price elasticity, the more the seasonality pattern or any of the variable cost rates impact on both the optimal (deseasonalized) demand value and profit level.

Before developing an efficient algorithm to compute the values $\left\{F_{n}^{i}(T): n=1 \ldots, T\right\}$, we first establish several important structural properties of these values.

Theorem 1 In a lotsizing problem with a horizon of $T$ periods and constant setup costs, let $z(n)$ denote the minimum cost if at most $n$ order periods are used. $z(n)$ is decreasing and convex.

Proof: Let $\tilde{z}(n)$ denote the minimum cost among all strategies, which place exactly $n$ orders, not necessarily in distinct periods, and let $\sigma(n)$ denote the optimal strategy which achieves the cost value $\tilde{z}(n)$. (To fulfill the requirement that exactly $n$ orders are to be placed, it may be optimal to place multiple orders in the same period, even though additional orders in any given period fail to be beneficial.) We show that $\tilde{z}(n)$ is convex. This implies that an integer $1 \leq n^{*} \leq T$ exists such that

$$
\tilde{z}(n)>\tilde{z}(n+1) \quad \forall n<n^{*}
$$

For all $n \leq n^{*}, \sigma(n)$ places orders in $n$ distinct periods. (If for some $n \leq n^{*}, \sigma(n)$ places multiple orders in the same period, a strategy $\sigma^{\prime}$ with cost value $z^{\prime}<\tilde{z}(n)$ can be obtained, which places $(n-1)$ orders, such that $\tilde{z}(n-1) \leq z^{\prime}<\tilde{z}(n)$, contradicting (19).). Thus:

$$
z(n)=\left\{\begin{aligned}
\tilde{z}(n) & , n \leq n^{*} \\
\tilde{z}\left(n^{*}\right) & , n>n^{*}
\end{aligned}\right.
$$


By $(19), z(n)$ is decreasing and convex if $\tilde{z}(n)$ is convex.

To show the latter, it suffices to show, for all $n_{1}, n_{2} \geq 1$ that $\tilde{z}\left(n_{1}+n_{2}\right)-\tilde{z}\left(n_{1}+1\right) \geq \tilde{z}\left(n_{2}\right)-\tilde{z}(1)$ $\Leftrightarrow 2 \tilde{z}(1)+\tilde{z}\left(n_{1}+n_{2}\right)-\tilde{z}\left(n_{1}+1\right) \geq \tilde{z}\left(n_{2}\right)+\tilde{z}(1) \Leftrightarrow \tilde{z}(1)-\tilde{z}\left(n_{1}+n_{2}\right) \leq\left[\tilde{z}(1)-\tilde{z}\left(n_{1}+1\right)\right]+\left[\tilde{z}(1)-\tilde{z}\left(n_{2}\right)\right]$

Let $\Theta=\left\{t_{1}=1, t_{2}, \ldots, t_{n_{1}+n_{2}}\right\}$ denote the set of order periods employed by the strategy $\sigma$ which achieves the minimum cost value $\tilde{z}\left(n_{1}+n_{2}\right)$ among all strategies employing $\left(n_{1}+n_{2}\right)$ orders. Let $\sigma^{\prime}$ denote the modified strategy, under which the last $\left(n_{2}-1\right)$ orders in $\Theta$ are eliminated and all demands in periods $\left\{t_{n_{1}+1}+1, \ldots, T\right\}$ are procured in period $t_{n_{1}+1}$ and let $z^{\prime}$ denote its cost value. Similarly, let $z^{\prime \prime}$ denote the cost of the ZIO strategy which employs the $n_{2}$ order periods $\left\{1, t_{n_{1}+2}, \ldots, t_{n_{1}+n_{2}}\right\}$. Note that:

$\tilde{z}(1)-\tilde{z}\left(n_{1}+n_{2}\right)=\left[\tilde{z}(1)-z^{\prime}\right]+\left[z^{\prime}-\tilde{z}\left(n_{1}+n_{2}\right)\right] \leq\left[\tilde{z}(1)-\tilde{z}\left(n_{1}+1\right)\right]+\left[z^{\prime}-\tilde{z}\left(n_{1}+n_{2}\right)\right]$ $\leq\left[\tilde{z}(1)-\tilde{z}\left(n_{1}+1\right)\right]+\left[\tilde{z}(1)-\tilde{z}\left(n_{2}\right)\right]$.

The first inequality is immediate since $\sigma^{\prime}$ is one feasible strategy employing $n_{1}+1$ orders. To verify the second inequality, let for all $1 \leq s<t \leq T$ :

$\tilde{C}_{s t} \quad=$ the difference in the variable cost of procuring a unit in period $s$ as opposed to period $t$, to satisfy demand in some period $t^{\prime} \geq t$

$=($ the variable order cost in period $\mathrm{s})-($ the variable order cost in period $\mathrm{t})+$ the cost of carrying a unit in inventory in periods $s, s+1, \ldots, t-1$

$d_{s t} \quad=$ aggregate demand in periods $s, s+1, \ldots, t-1$

$K \quad=\quad$ fixed order cost

Note that:

$$
\begin{aligned}
& \tilde{C}_{r s}+\tilde{C}_{s t}=\tilde{C}_{r t} \quad \forall r<s<t \\
& \tilde{C}_{t_{\ell} t_{\ell+1}} \geq 0 \quad \forall \ell=1, \ldots, n_{1}+n_{2}-1
\end{aligned}
$$

since, otherwise, a cost reduction could be achieved by moving the entire order at time $t_{\ell+1}$ forward to period $t_{\ell}$, as an additional order beyond the order(s) already scheduled for that period. This would contradict the optimality of the strategy $\sigma$. Thus, with $t_{n_{1}+n_{2}+1}=T$ : 


$$
\begin{aligned}
& z^{\prime}-\tilde{z}\left(n_{1}+n_{2}\right)=\sum_{\ell=n_{1}+2}^{n_{1}+n_{2}} \tilde{C}_{t_{n_{1}+1}, t_{\ell}} d_{t_{\ell} t_{\ell+1}}-\left(n_{2}-1\right) K \leq \sum_{\ell=n_{1}+2}^{n_{1}+n_{2}}\left[\tilde{C}_{1 t_{n_{1}+1}}+\tilde{C}_{t_{n_{1}}+1, t_{\ell}}\right] d_{t_{\ell} t_{\ell+1}}- \\
& \left(n_{2}-1\right) K=\sum_{\ell=n_{1}+2}^{n_{1}+n_{2}} \tilde{C}_{1 t_{\ell}} d_{t_{\ell} t_{\ell+1}}-\left(n_{2}-1\right) K=\tilde{z}(1)-z^{\prime \prime} \leq \tilde{z}(1)-\tilde{z}\left(n_{2}\right),
\end{aligned}
$$

where the first inequality follows from (22), the second equality from (21) and the last inequality from the fact that $z^{\prime \prime}$ is the cost of some feasible strategy employing $n_{2}$ orders, while $\tilde{z}\left(n_{2}\right)$ is the minimum cost among all such strategies.

Since $F_{n}^{i}(t)$ represents the cost value of a dynamic lot sizing problem with constant, in fact zero, setup costs, it follows that the values $\left\{F_{n}^{i}(t): n=1, \ldots, T\right\}$ are decreasing and convex.

\subsection{An optimal lot sizing $O\left(T^{2}\right)$ procedure with a given number of orders}

In this Subsection, we develop an $O\left(T^{2}\right)$-procedure to compute the values $\left\{F_{n}^{i}(T): n=1, \ldots, T\right\}$, defined in (11), and needed in the evaluation of (12). The procedure computes all entries of the ma-

$\operatorname{trix} F \stackrel{\text { def }}{=}\left\{F_{n}^{i}(t): n=1, \ldots, T ; t=1, \ldots, T\right\}$, row by row, starting with the values $\left\{F_{1}^{i}(t): t=1, \ldots, T\right\}$ in the first row. Recall that $F_{n}^{i}(T)$ denotes the minimal cost in a dynamic lot sizing problem in which exactly $n$ orders are to be placed. To simplify the expressions we drop the superscripts in this subsection. We first need the following notation:

$B(t)=\sum_{k=1}^{t} \beta_{k}=$ the cumulative demand factor over the first $t$ periods.

$c_{k l}=c_{k}+h_{k}+\cdots+h_{l-1}=$ the variable cost of procuring a unit in period $k$ and maintaining it in inventory until period $l(k<l)=c_{k, l-1}+h_{l-1}$

$H(t)=\sum_{k=1}^{t} h_{k}=$ the cost of carrying a unit of in stock from period 1 until the beginning of period $t+1=H(t-1)+h_{t}$

$S(k, t)=$ the total inventory carrying cost in periods $k, k+1, \ldots, t$ when placing an order in period $k$ to meet the cumulative demand in periods $k, \ldots, t$.

$s(t) \quad=\quad S(1, t)=s(t-1)+\beta_{t} H(t-1)$.

$\tilde{C}(k)=c_{k T}-h_{1 T}=c_{k}-H(k-1)$.

(For all $k<l, \tilde{C}(l)-\tilde{C}(k)$ is the additional cost of procuring a unit in period $l$ as opposed to procuring it in the earlier period $k$ and keeping it in stock until period l.)

Note that the first row of the matrix $F$, i.e. the values $\left\{F_{1}(t): t=1, \ldots, T\right\}$ are easily obtained 
in $O(T)$ time from the recursion:

$$
F_{1}(1)=c_{1} \beta_{1} ; \quad F_{1}(t)=F_{1}(t-1)+c_{1} \beta_{t}+\beta_{t} H(t-1), t=2 \ldots, T
$$

Assume therefore that the first $(n-1)$ rows of $F$ have been calculated. We now show how the $n-$ th row can be determined in $O(T)$-time by a 'list-based' procedure, similar to that in FEDERGRUEN and Tzur (1991) for the unrestricted lot sizing problem. To this end, let for all $t=1, \ldots, T$ :

$F_{n}(l, t)=$ the minimum cost in periods $1, \ldots, t$, under exactly $n$ orders, when period $l$ is the last order period preceding period $t$.

To decide whether for a given horizon $t$, some period $l$ is a better 'last' order period than some earlier period $k<l$, consider the difference function $\Delta_{n, k, l}(t) \stackrel{\text { def }}{=} F_{n}(k, t)-F_{n}(l, t)$ and note:

$$
\begin{aligned}
F_{n}(l, t) & =F_{n-1}(l-1)+S(l, t)+c_{l}[B(t)-B(l-1)] \\
F_{n}(k, t) & =F_{n-1}(k-1)+S(k, t)+c_{k}[B(t)-B(k-1)]
\end{aligned}
$$

Subtracting (24) from (25), we obtain after some algebra that

$$
\begin{aligned}
& \Delta_{n, k, l}(t)=A_{n}(k, l)+[\tilde{C}(k)-\tilde{C}(l)] B(t) \quad, \quad \text { where } \\
& A_{n}(k, l)=F_{n-1}(k-1)-F_{n-1}(l-1)+s(l-1)-s(k) \\
& +\tilde{C}(k)[B(l-1)-B(k-1)]+\beta_{k} H(k-1)+B(k-1)(\tilde{C}(l-1)-\tilde{C}(k))
\end{aligned}
$$

Thus, the difference function $\Delta_{n, k, l}(t)$ depends on the cost and demand parameters in the periods $l+1, \ldots, t$ only via the single characteristic $B(t)$, i.e. the cumulative demand factor in periods $1, \ldots, t$. Moreover the difference function is an affine function of $B(t)$, with $A_{n}(k, l)$ its intercept. Furthermore, it is easy to characterize for which values $B(t), k$ dominates $l$ as a last order period. Consider the following two cases:

(I) $\tilde{C}(k) \leq \tilde{C}(l)$ : The condition is equivalent to $c_{k, l} \leq c_{k}$; in this case, it is never strictly better to use $l$ as the last order period rather than an earlier period. To show this, consider an optimal ZIO plan for some planning horizon $t \geq l$, with $l$ as the last order period. Since $c_{k, l} \leq c_{l}$, costs do 
not increase when placing the order in the earlier period $k$. The resulting policy may fail to be a ZIO policy but via a series of perturbations it can be transformed into a ZIO policy of equal or lower cost with the same set of order periods, i.e. with a period before $l$ as the last order period.

(II) $\tilde{C}(k)>\tilde{C}(l)$ : In this case, it follows from (26) that $l$ is a strictly better last order period than $k$ if and only if

$$
B(t)>R_{n}(k, l) \stackrel{\text { def }}{=} A_{n}(k, l) /[\tilde{C}(k)-\tilde{C}(l)]
$$

Since each of the values in $\left\{F_{n}(t): t=1, \ldots, T\right\}$ represents the cost of a ZIO policy, these values are completely characterized by determining for all $t=1, \ldots, T$ :

$l_{n}(t)=$ the optimal last order period for the time interval $[1, t]$, when the total number of orders in this interval must equal $n$, i.e.

$$
F_{n}(t)=F_{n}\left(l_{n}(t), t\right)=\min _{l \leq t} F_{n}(l, t)
$$

(If more than one period qualifies as an optimal last order period, define $l_{n}(t)$ as the smallest such period). Clearly $F_{n}(t)=\infty$, if $t<n$, so we can restrict ourselves to the case where $t \geq n$.

To construct the list $\left\{l_{n}(t): t=1, \ldots, T\right\}$, we iteratively for $j=n, \ldots, T$, update a list $\mathcal{L}_{n}(j)=$ $\{i: n \leq i \leq j: i$ is the (lowested indexed) best last order period for some planning horizon $t>j$, with potential cumulative demand factor $B(t) \geq B(j)\}$, along with an ascending list of critical cumulative demand values $\{B(j)=r(1)<r(2)<\cdots<r(m)\}$, with $m=\left|\mathcal{L}_{n}(j)\right|$. The $k$-th element of the list $\mathcal{L}_{n}(j)$ is the best last order period (under the restriction of $n$ orders) for any horizon $t \geq j$ with cumulative demand $r(k) \leq B(t) \leq r(k+1), k=1, \ldots, m$. (Here, $r(m+1)=\infty$.) Thus, under the restriction of $\mathrm{n}$ orders, $\mathcal{L}_{n}(j)$ is a list containing all periods among the first $j$ periods, which may arise as an optimal last order period for some horizon $t>j$. In constructing this list, we treat all future demand factors $\beta_{j+1}, \ldots, \beta_{T}$ as unknown.

Clearly, the list $\left\{l_{n}(j): j=1, \ldots, T\right\}$ of actual optimal last order periods is simply the list of the first elements of the respective lists $\left\{\mathcal{L}_{n}(1), \mathcal{L}_{n}(2), \ldots, \mathcal{L}_{n}(T)\right\}$.

The following Algorithm summarizes the full procedure to determine the complete matrix $F=$ $\left\{F_{n}(t): t \leq n=1, \ldots, T ; t=1, \ldots, T\right\}$

We maintain at each iteration an ordered list $\mathcal{L}=(\{N[F I R S T], N[F I R S T+1], \ldots, N[L A S T]\})$ 
such that for $j=1, \ldots, T, \mathcal{L}=\mathcal{L}_{n}(j)$ at the end of the $\mathrm{j}$-th iteration. The records in this list are numbered FIRST, FIRST+1, ., LAST for appropriate values of FIRST and LAST. The $k$-th record $(F I R S T \leq k \leq L A S T)$ contains two numbers $\{N[k], r(k)\}$.

As explained in Lemma 2, below, periods are eliminated from either the front or the tail of the list $\mathcal{L}_{n}(\cdot)$. We therefore distinguish between two elementary procedures:

(i) DELTOP: this procedure deletes the first record of the list and sets FIRST := FIRST $=1$;

(ii) DELBOT: the procedure deletes the last record of the list and sets LAST := LAST-1;

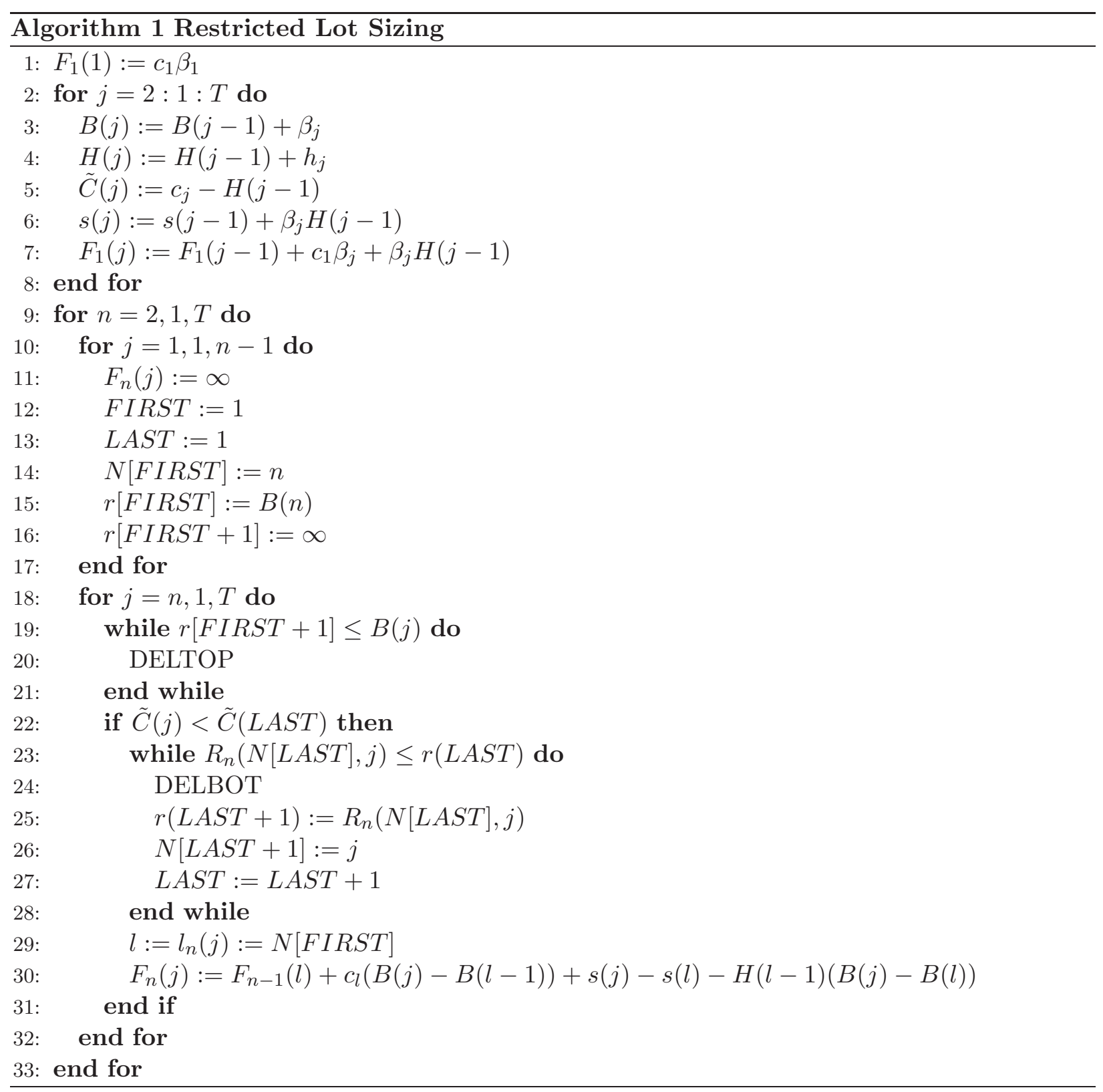


The computation of each row in the matrix F, i.e. the effort expended in the outer 'for do' loop in Step 1 is $O(T)$. (The procedures DELTOP and DELBOT are invoked at most $(\mathrm{T}-\mathrm{n}+1)$ times during this loop, since each period in $\{n, \ldots, T\}$ can be eliminated at most once, either by DELTOP or DELBOT. Both procedures are of complexity $O(1)$ as is the computation of the roots $R_{n}(1,1)$ via $\left.(28)\right)$. We conclude that the Algorithm requires $O\left(T^{2}\right)$ operations.

The following lemma identifies an effective way to 'update' the lists $\left\{\mathcal{L}_{n}(j): j=1, \ldots, T\right\}$. Clearly, $\mathcal{L}_{n}(n)=\{n\}$ with $r(n)=B(n)$.

Lemma 2 Fix $j=1, \ldots, T$ and let $\mathcal{L}_{n}(j)=\left(i_{1}, \ldots, i_{m}\right)$

(a) The periods in the list $\mathcal{L}_{n}(j)$ are distinct.

(b) $\mathcal{L}_{n}(j+1) \subseteq \mathcal{L}_{n}(j) \cup\{j+1\}$

(c) The periods appear in the list $\mathcal{L}_{n}(j)$ in descending order of their $\tilde{C}$ values, i.e. $\tilde{C}\left(i_{1}\right)>\tilde{C}\left(i_{2}\right)>\cdots>\tilde{C}\left(i_{m}\right)$

(d) If $\tilde{C}(j+1) \geq \tilde{C}\left(i_{m}\right)$, then the list $\mathcal{L}_{n}(j+1)=\mathcal{L}_{n}(j)$ and the critical values $r(1), \ldots, r(m)$ remain unchanged as well.

(e) If $\tilde{C}(j+1)<\tilde{C}\left(i_{m}\right)$, period $j+1$ enters at the bottom of the list. To determine the corresponding $r(\cdot)$-value, as well as which of the existing elements of the list are to be removed, compute sequentially for $k=m, m-1, \ldots 1$ the root $r^{*}=R_{n}\left(i_{k}, j+1\right)$ and eliminate period $i_{k}$ from the list as long as $r^{*} \leq r(k)$. The last computed root $r^{*}$ has $r^{*}>r(k)$ and is the $r(\cdot)$-value for period $j+1$.

Proof. (a) Assume, to the contrary, that some period $i$ appears more than once in the list, i.e. it is the best order period in two distinct intervals for the cumulative demand factor $B(t)$, but, for some inbetween values of $B(t)$, some other period $k$ is a better last order period. This implies that the difference function $\Delta_{n, k, l}(t)$, viewed as a function of $B(t)$, switches signs at least twice. Since the function is linear in $B(t)$ this results in a contradiction.

(b) Immediate.

(c) - (e): These parts are proven by induction with respect to $j=1, \ldots, T$. Assume therefore that for the $j$-th list $\mathcal{L}_{n}(j), \tilde{C}\left(i_{1}\right)>\tilde{C}\left(i_{2}\right)>\cdots>\tilde{C}\left(i_{m}\right)$. If $\tilde{C}(j+1) \geq \tilde{C}\left(i_{m}\right)$, we have shown 
under (I) above, that period $i_{m}$ is a better last order period than period $j+1$ for any future horizon $t>j$. This proves part $(\mathrm{d})$. Since the list remains unaltered, its elements continue to be ordered in decreasing order of their $\tilde{C}()$-values. If $\tilde{C}(j+1)<\tilde{C}\left(i_{m}\right), \tilde{C}(j+1)$ is smaller than the $\tilde{C}(\cdot)$-value of all elements of the list $\mathcal{L}_{n}(j)$, so that $(j+1)$ is the best last order period among all periods $1, \ldots, j+1$ for $B(t)$-values that are sufficiently large. This implies that $(j+1)$ is to be added to the list and since, by part (a), it enters in the list only once, it enters at the bottom of the list. This implies that the elements of the list $\mathcal{L}_{n}(j+1)$ continue to be ranked in decreasing order of their $\tilde{C}(\cdot)$-values, regardless of what elements of the list need to be eliminated, if any. This completes the induction proof for part (c). To verify the validity of the list-updating procedure in part (e), assume first that $r^{*}=R_{n}\left(i_{m}, j+1\right)>r(m)$. For $B(t) \geq r^{*}$, period $(j+1)$ is a better last order period than $i_{m}$ and the list $\mathcal{L}_{n}(j)$ reveals that the latter dominates all other periods in $\{1, \ldots, j\}$ on this half line. Thus, for $B(t)>r^{*}$, period $(\mathrm{j}+1)$ is the best last order period among all of the first $(\mathrm{j}+1)$-periods. On the other hand, for $r(m) \leq B(t) \leq r^{*}$, period $i_{m}$ dominates period $(\mathrm{j}+1)$ as well as all periods in $\{1, \ldots, j\}$, so it is the best last order period among the first $(\mathrm{j}+1)$-periods. It is also easily verified that each of the previous elements $i_{k}, k<m$ in the list continues to dominate on the interval $[r(k), r(k+1)]$ even when considering period $(\mathrm{j}+1)$ as an alternative. We conclude that, in this case, the list $\mathcal{L}_{n}(j+1)$ is obtained from the list $\mathcal{L}_{n}(j)$, simply by appending period $(j+1)$ to its tail with $g(m+1)=r^{*}$.

Consider now the remaining case where $r^{*}=R_{n}\left(i_{m}, j+1\right)<r(m)$. In this case, period $i_{m}$ is dominated by period $(j+1)$ for $B(t) \geq r(m)$, while it is dominated by some period in $\{1, \ldots, j\}$ for $B(t)<r(m)$. This implies that period $i_{m}$ is to be eliminated from the list and the updating process can now proceed with this curtailed list.

Remark: Upon completion of the list $\mathcal{L}_{n}(j+1)$, it is advisable to consider the actual cumulative demand factor value $B(j+1)$ and eliminate from the front of the list all elements with an $r(\cdot)$-value below $B(j+1)$. (After all, all future horizons $t>j+1$ have $B(t) \geq B(j+1)$.)

Example 3: (Linear Demand Functions) Consider an industry with $N=3$ firms and desea- 
sonalized demand functions:

$$
\begin{aligned}
& \delta_{1}(p)=400-10 p_{1}+p_{2}+p_{3} \\
& \delta_{2}(p)=250+p_{1}-12 p_{2}+10 p_{3} \\
& \delta_{3}(p)=250+p_{1}+10 p_{2}-12 p_{3}
\end{aligned}
$$

The firms face a planning horizon of $T=54$ periods. We consider six different seasonality patterns $\left\{\beta_{t}: t=1, \ldots, 54\right\}$, which satisfy $(2)$ and are common to all three firms, as follows:

(I) (Time-invariant demand functions) $\beta_{t}=1 ; t=1, \ldots, 54$

(II) (Linear Growth) $\beta_{t}=0.25+1.5 \frac{(t-1)}{53} ; t=1, \ldots, 54$

(III) (Linear Decline) $\beta_{t}=1.75-1.5 \frac{(t-1)}{53} ; t=1, \ldots, 54$

(IV) (Holiday Season at Beginning of Planning Horizon)

$$
\beta_{t}=\left\{\begin{aligned}
\frac{54}{114}+\frac{540}{570}(t-1) & , t=1, \ldots, 6 \\
\frac{594}{114}-\frac{540}{570}(t-7) & , t=7, \ldots, 12 \\
\frac{54}{114} & , t=13, \ldots, 54
\end{aligned}\right.
$$

(V) (Holiday Season at End of planning Horizon)

$$
\beta_{t}=\left\{\begin{aligned}
\frac{54}{114} & , t & =1, \ldots, 42 \\
\frac{54}{114}+\frac{540}{570}(t-43) & , t & =43, \ldots, 48 \\
\frac{594}{114}-\frac{540}{570}(t-49) & , t & =49, \ldots, 54
\end{aligned}\right.
$$

(VI) (Cyclical Pattern)

$$
\beta_{t}=\left\{\begin{aligned}
0.25+0.75(t-1) & , t=1, \ldots, 3 \\
1.75-0.75(t-4) & , t=4, \ldots, 6 \\
\beta_{t \bmod 6} & , t=7, \ldots, 54
\end{aligned}\right.
$$

where $\mathrm{t}$ mod 6 denotes t modulo 6 . The first pattern reflects a situation where demand functions are time-invariant and the second (third) pattern one with linear growth (decline). The fourth and fifth patterns represent a planning horizon with a single season of peak demands either at the beginning or at the end of the planning horizon. The last pattern (VI) is cyclical with a cycle 

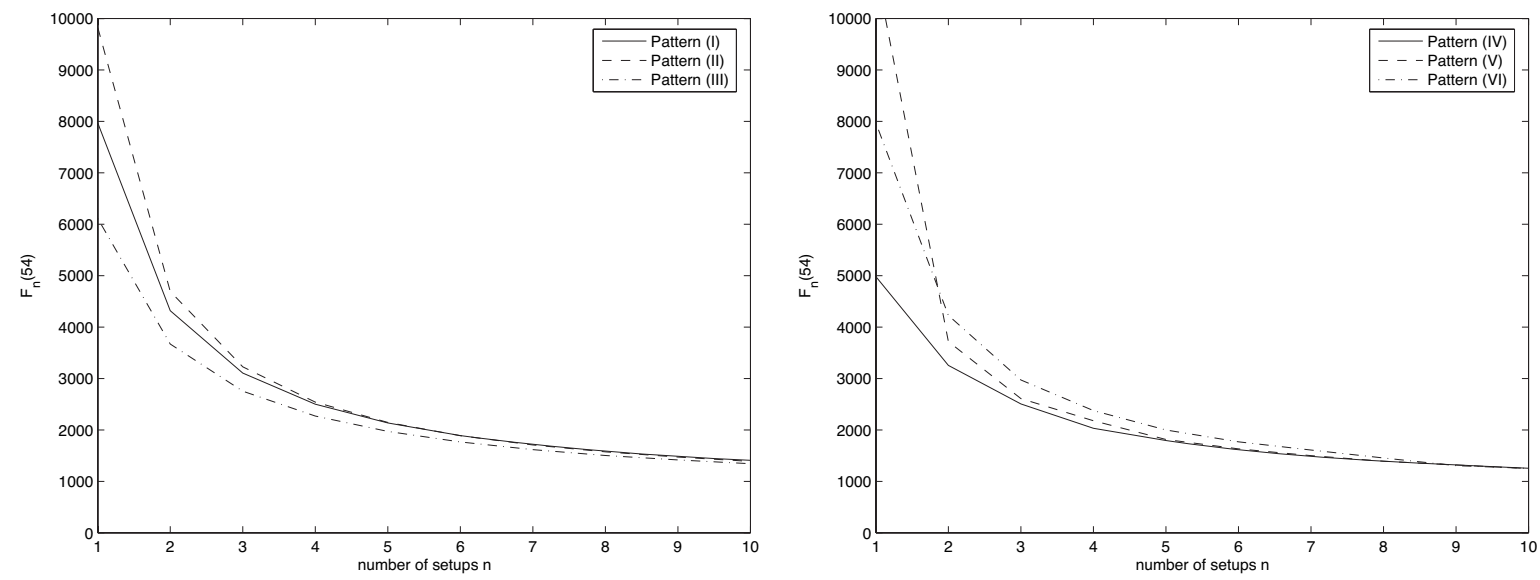

Figure 1(a) and 1(b): $F_{n}(54)$ as a function of the number of setup periods $\mathbf{n}$

length of 6 periods, such that demands in the two middle periods of each cycle are 7 times their value in the first and last period, while $\beta_{t}=1$ in the remaining two periods of the cycle.

All three firms share the same cost parameters which are time-invariant: $K_{t}^{i}=1000 ; \quad c_{t}^{i}=$ $15 ; \quad h_{t}^{i}=5$ for all $i=1, \ldots, 3 ; \quad t=1, \ldots, 54$. Since the firms have identical $c$ - and $h$ values and the firms have identical seasonality patterns, they also share the same values for $\left\{F_{n}^{i}(t): t=1, \ldots, 54\right\}$. In Figure $1(\mathrm{a})$ and $1(\mathrm{~b})$ we display the values $\left\{F_{n}(54)\right\}$ as a function of the permitted number of setup periods $n=1, \ldots, 54$, for the six seasonality patterns (I)-(VI). Observe that the $F_{n}(54)$-values are significantly different across the six patterns, for small numbers of permitted setup periods, but the relative difference gradually decreases as $n$ increases. Since the parameters $c_{t}^{i}$ parameters are constant over time, the variable procurement cost component in $\left\{F_{n}(54)\right\}$ is identical for all $n=1, \ldots, 54$ and for all seasonality patterns (I)-(VI). All differences in the $\left\{F_{n}(54)\right\}$ values are therefore attributable to differences in the holding costs. These, of course, decline to zero as $n$ increases to 54 . For all $n \geq 7$, the cyclical pattern (VI) is the least expensive to service and the time-invariant pattern (I) the most expensive. This contradicts common folklore which assumes that optimal costs are achieved when one is facing a smooth, time-invariant sales pattern.

Theorem 1 shows that the curves $\left\{F_{n}(54): n=1, \ldots, 54\right\}$ are decreasing and convex. Indeed, 


\begin{tabular}{rrrrr}
\hline pattern & $\gamma$ & $\eta$ & $\zeta$ & relative gap \\
\hline$(\mathrm{I})$ & 1.0 & 687.1 & 7268.1 & $0.58 \%$ \\
$(\mathrm{II})$ & 1.2 & 769.1 & 9090.8 & $2.18 \%$ \\
$(\mathrm{II})$ & 0.9 & 654.5 & 5513.9 & $0.44 \%$ \\
$(\mathrm{IV})$ & 0.8 & 591.6 & 4422.2 & $2.45 \%$ \\
$(\mathrm{~V})$ & 1.6 & 867.3 & 10012.6 & $6.16 \%$ \\
$(\mathrm{VI})$ & 1.1 & 713.1 & 7330.9 & $0.94 \%$ \\
\hline
\end{tabular}

Table 1: Approximating curves for patterns (I) to (VI)

they can be approximated very closely by curves of the shape

$$
F_{n}^{i}(T) \sim T\left[\eta^{i}+\frac{\zeta^{i}}{n^{\gamma_{i}}}\right] \quad, \quad n=1, \ldots, T
$$

for appropriate constants $\eta^{i}, \zeta^{i}>0$ and $0<\gamma_{i}<2$. For example, these constants can be chosen to minimize the sum of squared differences between the left and the right sides of (36). Table 1 below exhibits the parameters generating the best possible fit:

The last column in Table 1 displays, for the six patterns, the average relative difference between the exact and the approximate curve. We have found that approximations of the type (36) are, in fact, very close, across the board, for any combination of cost values and seasonality patterns.

We now turn to the best response problem firm 1 faces when both of his competitors choose a price value of $\$ 30$. Based on (14) and (15), Figure 2 exhibits the optimal profit $\pi^{* 1}(n)$ for pattern (I) and (VI) as a function of the permitted number of setups $n$. The globally optimal prices which solve the best response problem are $\$ 31.30$ and $\$ 30.89$ and correspond with a lot sizing schedule with $n=33$ for pattern (I) and $n=35$ for pattern (VI).

Example 4: (Cobb Douglas Demand Functions) In this example, we utilize the following Cobb-Douglas demand functions:

$$
\begin{aligned}
& \delta^{1}=80000\left(p^{1}\right)^{-1.875}\left(p^{2}\right)^{0.1875}\left(p^{3}\right)^{0.1875} \\
& \delta^{2}=2000\left(p^{2}\right)^{-1.636}\left(p^{1}\right)^{0.136}\left(p^{3}\right)^{1.363} \\
& \delta^{3}=2000\left(p^{3}\right)^{-1.636}\left(p^{1}\right)^{0.136}\left(p^{2}\right)^{1.363}
\end{aligned}
$$

At the price vector $p=\{30,30,30\}$, this set of demand functions has the same direct price 


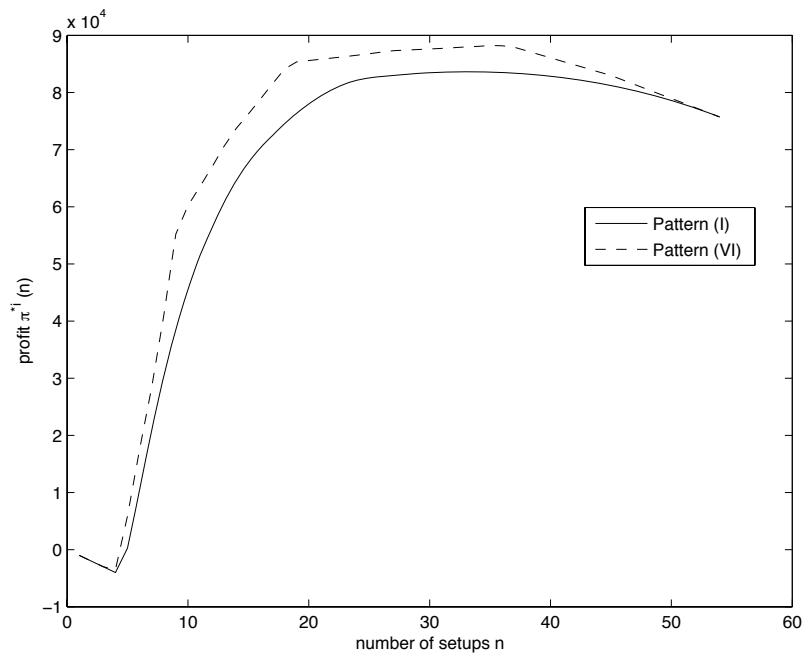

\section{Figure 2: (Linear Demand Functions) Optimal profit for firm 1 as a function of permitted number of setups; pattern (I) and (VI) when $K_{t}^{i}=1000$}

elasticities as the linear functions in Example 3. (Recall, for Cobb-Douglas demand functions, these elasticities are constant and they are given by the exponents of the price factors in these functions, the elasticities fail to be constant for linear demand functions.) The multiplicative constants are chosen to exemplify several properties of the equilibrium behavior, see $\S 5$.

We, again, address the best response problem firm 1 faces when both of his competitors choose a price of $\$ 30$ and $K=5000$. Based on (18), Figure 3 again exhibits the optimal profit $\pi^{* i}(n)$ for patterns (I) and (VI), as a function of the permitted number of orders $n$. Under pattern (I) ((VI)), the optimal price is $\$ 40.83$ (\$39.63) and the optimal number of orders 20 (18). (When $\mathrm{K}=1000$, it is optimal to place an order in every period under both patterns.)

\section{The Equilibrium Analysis}

In this $\S$, we analyze the equilibrium behavior in the industry under price - or Bertrand competition. (See however, the end of this section for a discussion of the case of Cournot competition.) As in $\S 3$, we assume, here, that the fixed order costs are constant throughout the planning horizon. Time-varying order costs are discussed in $\S 6$, along with other generalizations.

Substituting the expression (10) for the cost function $C^{i}(p)$ as well as the identity $d_{t}^{i}(p)=\beta_{t}^{i} \delta^{i}(p)$ 


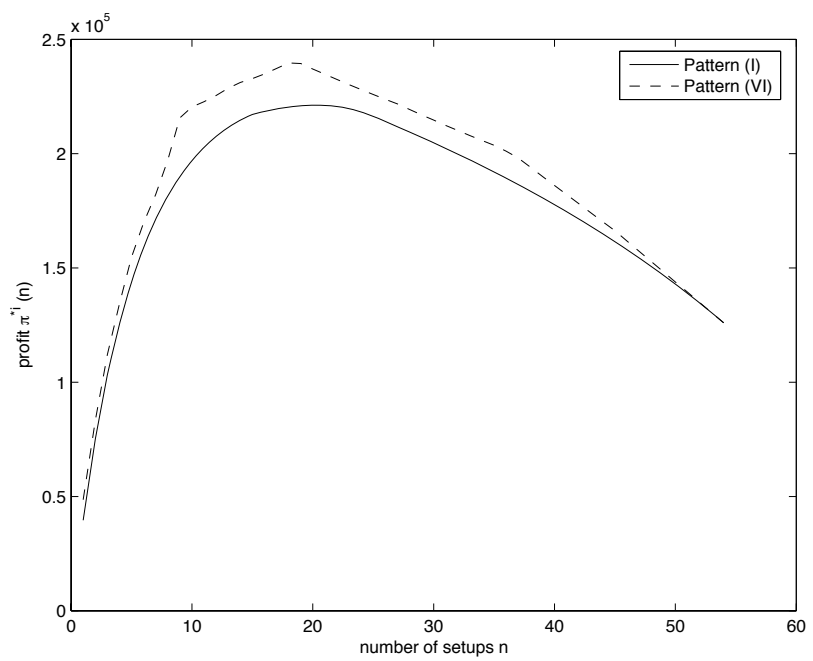

Figure 3: (Cobb Douglas Demand Functions) Optimal profit for firm 1 as a function of permitted number of setups; pattern (I) and (VI) when $K_{t}^{i}=5000$

in the profit function (18), we obtain:

$$
\pi^{i}(p)=T p^{i} \delta^{i}(p)-\min _{n=1, \ldots, T}\left\{n K^{i}+T \underline{\mathrm{c}}^{i} \delta^{i}(p)+\delta^{i}(p) \tilde{F}_{n}^{i}(T)\right\}
$$

where, with $\underline{\mathrm{c}}^{i}=\min _{t=1, \ldots, T} c_{t}^{i}, \quad i=1, \ldots, N$ :

$$
\tilde{F}_{n}^{i}(T)=F_{n}^{i}(T)-T \underline{\mathrm{c}}^{i}
$$

A sufficient condition for the existence of a price equilibrium is the well-known Nash-Debreu condition:

(C) Firm i's profit function $\pi^{i}(p)$ is quasi-concave in its own price $p^{i}$ for all $i=1, \ldots, N$.

While the revenue term in (38) is concave in $p^{i}$, the cost term is (piecewise linear) concave, as well, adding a convex term to the profit function. Below we identify an intuitive condition which guarantees that the concavity of the revenue term 'dominates' the convexity effect of the cost function. As the condition relates the price elasticity to the turnover rate, i.e. the sales to inventory ratio, we proceed to show that it is almost invariably satisfied.

Since the cost function in (38) is a piecewise linear concave function of the deseasonalized 
demand volume, it is difficult to use this representation to verify when condition (C) is satisfied. However, the following provides a very close approximation of this cost function via a single closed form differentiable function:

By Theorem $1, \tilde{F}_{n}^{i}(T)$ is convex and decreasing in $n$. Let $\Phi^{i}(x)$ be a convex and differentiable extension of the $\left\{\tilde{F}_{n}^{i}(T): n=1,2, \ldots\right\}$ curve, i.e. $\Phi^{i}(n)=\tilde{F}_{n}^{i}(T)$ for all $n=1,2, \ldots$ The following is, therefore, a close upper bound approximation of the profit function $\pi^{i}$ :

$$
\begin{aligned}
\tilde{\pi}^{i}(p) & =T \delta^{i}(p)\left(p^{i}-\underline{c}^{i}\right)-\min _{x \geq 0}\left\{x K^{i}+\delta^{i}(p) \Phi^{i}(x)\right\} \\
& =T \delta^{i}(p)\left(p^{i}-\underline{c}^{i}\right)-\left[K^{i} \varphi^{i}\left(\frac{-K^{i}}{\delta^{i}(p)}\right)+\delta^{i}(p) \Phi^{i}\left(\phi^{i}\left(\frac{-K^{i}}{\delta^{i}(p)}\right)\right)\right]
\end{aligned}
$$

where $\varphi^{i}(u)=\left(\Phi^{i^{\prime}}\right)^{-1}(u)$. Convexity of this function can be verified directly by inspecting the sign of $\delta^{2} \tilde{\pi}^{i}(p) /\left(\partial p^{i}\right)^{2}$. An alternative approximation is obtained by replacing $\tilde{F}_{n}^{i}(T)$ by a function of the form (36) and treating $n$ as a continuous variable:

$$
\tilde{\pi}^{i}(p)=T \delta^{i}\left(p^{i}-\underline{\mathrm{c}}^{i}-\eta^{i}\right)-\min \left\{n K^{i}+\frac{\zeta^{i} \delta^{i}(p)}{n^{\gamma^{i}+1}}: n \in \mathbb{R}\right\}
$$

The convex minimization problem to the right of (42) can be solved in closed form by setting the first derivative of the minimand equal to zero. After some algebra this results in:

$$
\tilde{\pi}^{i}(p)=T \delta^{i}(p)\left(p^{i}-\underline{\mathrm{c}}^{i}-\eta^{i}\right)-D \delta_{i}(p)^{\left(\gamma^{i}+1\right)^{-1}}
$$

where $D=\left(K^{i}\right)^{\gamma^{i} /\left(\gamma^{i}+1\right)}\left(\gamma^{i} \eta^{i}\right)^{\left(\gamma^{i}+1\right)^{-1}}$. In other words, the non-linear part of the cost function in (43) can be represented as a simple concave power function of the deseasonalized demand volume. This representation allows for a simple representation of the concavity condition $(\mathrm{C})$ for the profit functions or the equivalent condition $\delta^{2} \tilde{\pi}^{i} /\left(\partial p^{i}\right)^{2} \leq 0$.

A price competition game in which each firm selects a price from a closed interval and with profit functions of the type (43) has been analyzed by CACHON and HARKER (2002) and Bernstein and Federgruen (2003). The former confine themselves to the case where the number of firms $N=2$. Without guaranteeing that an equilibrium exists, they establish a sufficient condition under which the existence of multiple equilibria can be excluded. Bernstein and FEDERGRuen (2003) confine themselves to the case of linear demand functions and $\gamma^{i}=1$ for all $i$; they show that the existence 
of an equilibrium can, indeed, not be guaranteed under completely arbitrary parameters. Following their analysis, we identify, however, for the case of linear deseasonalized demand functions, a simple sufficient condition for the existence of an equilibrium, which relates the demand elasticity of a firm with respect to its own price to the firm's inventory-to-sales ratio. More specifically, assume the demand functions $\left\{\delta^{i}(p)\right\}$ satisfy (3). Let

$I N V^{i}=D \delta^{i}(p)^{\left(\gamma^{i}+1\right)^{-1}}=$ firm i's optimal inventory and fixed order costs over the course of the planning horizon, under the price vector p.

$R E V^{i}=T p^{i} \delta^{i}(p)=$ firm i's total gross revenue over the course of the planning horizon, under the price vector $\mathrm{p}$.

Theorem 2 below shows that a Nash equilibrium exists in the price competition model if the following easily interpretable condition is satisfied:

$$
\text { (C1) } \quad \epsilon_{i i} \leq 8 \frac{R E V^{i}}{I N V^{i}} \quad, \quad i=1, \ldots, N
$$

The proof of Theorem 2 shows that $(\mathrm{C} 1)$ is in fact equivalent to $(\mathrm{C})$, i. e. to the overall profit function of each firm being quasi-concave in its own price. (It is well known that games may fail to have a (pure) equilibrium if the players' profit functions fail to have this quasi-concavity or related structural properties, for example supermodularity, discussed below.)

The ratio $R E V^{i} / I N V^{i}$ is closely related to the (annual) sales-to-inventory ratio, one of Wall Street's most frequently monitored company measures. BERnSTEIN and FEDERGRUEN (2003) argue that the ratio $R E V^{i} / I N V^{i}$ is, in fact, at least 2.5 times the sales-to-inventory ratio. Moreover, analyzing data by DUN and BRADSTREeT (2001), the authors show for a sample of 10 consumer product lines that the average lower quartile of the sales-to-inventory ratio varies between 2.8 and 6.7 for the 10 product lines. As a consequence, the right hand side of the inequality in $(\mathrm{C} 1)$ varies between 56 and 134, while the absolute value of the price elasticity varies between one and five, see e.g. Tellis (1988). Thus, condition (C1) is very comfortably satisfied for virtually all industries.

While condition (C1) guarantees that a Nash equilibrium exists, two important questions remain: (a) Is the Nash equilibrium unique or can multiple equilibria arise, making it hard to predict which of the possible equilibria the industry will adopt and (b) How can the equilibrium (or equilibria) be computed efficiently? We show that under a slight tightening of condition $(\mathrm{C} 1)$, a unique 
equilibrium can indeed be guaranteed and that this unique equilibrium can be efficiently computed as the limit point of the following simple (iterative) tatônnement scheme:

Tatônnement scheme: Starting with an arbitrary price vector $p_{(0)}$, in the k-th iteration of the scheme, each firm determines the price $p_{(k)}^{i}$ which solves the best response problem (8), assuming all competing firms' prices are set according to their value in the price vector $p_{(k-1)}$.

Convergence of this tatônnement scheme is guaranteed when the game is supermodular. The proof of Theorem 2(b) shows that the supermodularity property is equivalent to a condition, closely related to $(\mathrm{C} 1)$ :

$$
\text { (C2) } \quad \epsilon^{i i} \leq 4 \frac{R E V^{i}}{I N V^{i}}
$$

As reviewed above, condition (C2), which is somewhat tighter than (C1), is still very comfortably satisfied for virtually all product lines.

Theorem 2 Assume the deseasonalized demand functions $\left\{\delta^{i}(p)\right\}$ are linear, i.e. they satisfy (3), while all fixed order costs are constant throughout time, i.e. $K_{1}^{i}=\ldots=K_{T}^{i}=K^{i}$. Consider the price competition game under the (approximate) profit functions $\tilde{\pi}^{i}(p)$ in (43).

(a) Under condition (C1), the price competition game has a Nash equilibrium

(b) Under condition (C2), the price competition game has a unique equilibrium $p^{*}$. The tatônnement scheme converges to $p^{*}$ from any starting point $p^{0}$.

Proof: (a) Note that

$$
\begin{aligned}
& \frac{\partial \tilde{\pi}^{i}}{\partial p^{i}}=T b^{i}\left(p^{i}-\underline{\mathrm{c}}^{i}-\eta^{i}\right)+T \delta^{i}(p)+\frac{b^{i}}{\gamma^{i}+1} D \delta^{i}(p)^{\frac{-\gamma^{i}}{\gamma^{i}+1}} \quad \text { and } \\
& \frac{\partial^{2} \tilde{\pi}^{i}}{\left(\partial p^{i}\right)^{2}}=-2 T b^{i}+D\left(b^{i}\right)^{2} \frac{\gamma^{i}}{\left(\gamma^{i}+1\right)^{2}}\left[\delta^{i}(p)\right]^{-\left(\frac{\gamma^{i}}{\gamma^{i}+1}+1\right)}
\end{aligned}
$$

Condition (C) for the existence of a Nash equilibrium is satisfied if and only if

$$
\frac{\partial^{2} \tilde{\pi}^{i}}{\left(\partial p^{i}\right)^{2}} \leq 0 \Leftrightarrow \epsilon^{i i}=\frac{b_{i} p_{i}}{\delta^{i}(p)} \leq 2\left[\gamma^{i}+2+\frac{1}{\gamma^{i}}\right] \frac{T p^{i} \delta^{i}(p)}{D\left(\delta^{i}\right)^{\left(\gamma^{i}+1\right)^{-1}}}=2\left[\gamma^{i}+2+\frac{1}{\gamma^{i}}\right] \frac{R E V^{i}}{I N V^{i}}
$$


Thus $(\mathrm{C} 1) \Leftrightarrow(\mathrm{C})$ since $8 \leq 2\left(\gamma^{i}+2+\frac{1}{\gamma^{i}}\right)$ as the function $g(x) \equiv x+\frac{1}{x}$ achieves its minimum value of 2 for $x=1$.

(b) For the tatônnement scheme to converge to a Nash equilibrium, it suffices to show that the profit function $\tilde{\pi}^{i}$ is supermodular in $\left(p^{i}, p^{j}\right)$ for all $i \neq j$, i.e. that

$$
\frac{\partial^{2} \tilde{\pi}^{2}}{\partial p^{i} \partial p^{j}}=T \theta_{j}^{i}-b^{i} \theta_{j}^{i} D \frac{\gamma^{i}}{\left(\gamma^{i}+1\right)^{2}}\left[\delta^{i}(p)\right]^{-\left(\frac{\gamma^{i}}{\gamma^{i}+1}+1\right)} \geq 0
$$

But, following the algebra in part (a), this inequality is equivalent to $\epsilon^{i i} \leq\left[\gamma^{i}+2+\frac{1}{\gamma^{i}}\right] \frac{R E V^{i}}{I N V^{i}}$, which is satisfied under (C2) for all $\gamma^{i}>0$ since, for all $\gamma^{i}>0, \gamma^{i}+\frac{1}{\gamma^{i}} \geq 2$, as shown in part (a). Finally, to show that the equilibrium is unique, it suffices to verify that

$$
\sum_{j \neq i} \frac{\partial^{2} \tilde{\pi}^{i}}{\partial p^{i} \partial p^{j}} \leq\left|\frac{\partial^{2} \tilde{\pi}^{i}}{\left(\partial p^{i}\right)^{2}}\right| \quad \forall \quad i=1, \ldots, N
$$

But $\sum_{j \neq i} \frac{\partial^{2} \tilde{\pi}^{i}}{\partial p^{i} \partial p^{j}}=\left(\sum_{j \neq i} \theta_{j}^{i}\right)\left[T-b^{i} \frac{D \gamma^{i}}{\left(\gamma^{i}+1\right)^{2}}\left(\delta^{i}(p)\right)^{-\left(\frac{\gamma^{i}}{\gamma^{i}+1}+1\right)}\right] \leq b^{i} T-\left(b^{i}\right)^{2} D \frac{\gamma^{i}}{\left(\gamma^{i}+1\right)^{2}}\left[\delta^{2}(p)^{-\left(\frac{\gamma^{i}}{\gamma^{i}+1}+1\right)}\right]=$ $\left|\frac{\partial \tilde{\pi}^{i}}{\left(\partial p^{i}\right)^{2}}\right|$ where the first inequality follows from (4) and (49) and the last one from (47).

The proof goes along the lines of those of Theorem 1 and 2 in BERnstein and Federgruen (2003). For specific classes of nonlinear demand functions, conditions of similar intuitive appeal as (C1) can be derived to guarantee that the profit function $\tilde{\pi}^{i}(p)$ is concave in $p^{i}$. For example, for the Cobb-Douglas functions (5), it can be shown that $\tilde{\pi}^{i}(p)$ is concave in $p^{i}$ if either the absolute price elasticity $b^{i}$ or $\left(p^{i, \max }-\underline{\mathrm{c}}^{i}-\eta^{i}\right)$ the maximum gross profit margin, is not too large.

The tatônnement scheme consists of repeated solutions of best response problems. The availability of an efficient procedure to solve the best response problems (see $\S 3$ ) is therefore of critical importance. Note that the $O\left(T^{2}\right)$ algorithm to compute the values $\left\{F_{n}^{i}(T)\right\}$ needs to be executed only once in the first iteration of the tatônnement scheme. Thereafter, in each additional iteration, the best response problem only requires $O(T)$ operations and evaluations of inverse derivative revenue function $\left(R_{i}^{\prime}\right)^{-1}(\cdot)$.

Consider now the case where the firms engage in Cournot (quantity) competition as opposed to Bertrand (price) competition. Consider, again, the conditions stated in Theorem 1, and assume the approximate profit functions $\tilde{\pi}^{i}(\cdot)$ in (43) are used. Under (3), the system of (deseasonalized) 
demand functions can be inverted resulting in the inverse demand functions:

$$
p^{i}=\hat{a}^{i}-\hat{b}^{i} \delta^{i}-\sum_{j \neq i} \hat{\theta}_{j}^{i} \delta^{j} \quad, \quad i=1, \ldots, N \quad \text { where } \quad \hat{b}^{i}, \hat{\theta}_{j}^{i} \geq 0
$$

It follows from a straightforward generalization of Theorem 3 in BERNSTEIN and FEDERGRUEN (2003) that an equilibrium exists under condition (C1) and that this equilibrium is unique under (C2) and the condition

$$
\hat{b}^{i}>\sum_{j \neq i} \hat{\theta}_{j}^{i} \quad, \quad i=1, \ldots, N
$$

the direct counterpart of (4) for inverse demand functions. Thus, the same condition (C1) guarantees an equilibrium both under price- and under quantity competition. Moreover, an analogous pair of conditions guarantees that the equilibrium is unique. However, in contrast to the case of price competition, it is no longer possible to ensure that the tatônnement scheme converges to an equilibrium, even when a unique equilibrium can be guaranteed.

\section{$5 \quad$ Numerical Examples}

In this $\S$, we report on a numerical study conducted to investigate the effect on the equilibrium behavior of seasonality patterns and economies of scale resulting from fixed setup costs.

Table 2 displays the equilibrium in our base example (Example 2) under each of the six seasonality patterns (I)-(VI) and four combinations of setup cost values: (a) $K_{t}^{i}=500$; (b) $K_{t}^{i}=1000$; (c) $K_{t}^{i}=4000$ and (d) $K_{t}^{i}=5400$. Each of the cells in the table corresponds with one of the 24 problem instances. Whenever a unique equilibrium exists, we display, sequentially, the equilibrium prices $\left(p^{*}\right)$, volumes $\left(\delta^{*}\right)$, profit levels $\left(\pi^{*}\right)$ and the number of order periods $\left(n^{*}\right)$ for each firm. (If no or multiple equilibria arise, the cell is left empty.)

In our example, firms 2 and 3 have identical characteristics. Firm 1 has a larger intercept as well as a significantly higher 'total price sensitivity' defined by $\bar{b}_{i}=b_{i}-\sum_{j \neq i} \theta_{j}^{i}>0$, see (4). (The total price sensitivity measures the marginal decline in sales volume due to a universal price increase in the industry; note $\bar{b}_{1}=8$ and $\bar{b}_{2}=\bar{b}_{3}=1$.) The larger total price sensitivity for firm 1 induces it, in all 24 problem instances considered, to adopt a lower price than his competitors, the 


\begin{tabular}{|c|c|c|c|c|c|c|c|}
\hline & & (I) & (II) & (III) & (IV) & $(\mathrm{V})$ & (VI) \\
\hline \multirow{4}{*}{$\mathrm{K}=500$} & $p^{*}$ & 30.7932 .9132 .91 & 30.9433 .0433 .04 & 30.9433 .0333 .03 & $31.2832 .95 \quad 32.95$ & $31.28 \quad 32.95 \quad 32.95$ & 31.1033 .1133 .11 \\
\hline & $\delta^{*}$ & 157.91214 .96214 .96 & 156.59214 .87214 .87 & 156.67214 .88214 .88 & 153.12215 .38215 .38 & 153.12215 .38215 .38 & 155.22214 .87214 .87 \\
\hline & $\pi^{*}$ & $107.66 \quad 180.94 \quad 180.94$ & $\begin{array}{llll}108.91 & 182.76 & 182.76\end{array}$ & $\begin{array}{llll}109.05 & 182.78 & 182.78\end{array}$ & $110.61 \quad 181.75 \quad 181.75$ & $110.61 \quad 181.75 \quad 181.75$ & $\begin{array}{llll}11 & 185.26 & 185.26\end{array}$ \\
\hline & $n^{*}$ & 545454 & 475050 & 475050 & 325454 & 325454 & 374545 \\
\hline \multirow{4}{*}{$\mathrm{K}=1000$} & $p^{*}$ & 32.0533 .0033 .00 & 31.6033 .6133 .61 & 31.6333 .5733 .57 & 31.3633 .7833 .78 & $31.4033 .78 \quad 33.78$ & 31.2433 .4433 .44 \\
\hline & $\delta^{*}$ & 145.50216 .04216 .04 & $151.20 \quad 214.39214 .39$ & $150.88 \quad 214.48 \quad 214.48$ & 153.96213 .80213 .80 & 153.52213 .84213 .84 & $154.51 \quad 214.35 \quad 214.35$ \\
\hline & $\pi^{*}$ & $87.33156 .04 \quad 156.04$ & $89.45 \quad 165.83 \quad 165.83$ & 89.93166 .00166 .00 & $95.99 \quad 173.69173 .69$ & $96.27 \quad 173.76 \quad 173.76$ & $\begin{array}{rlll}93.91 & 169.76 & 169.76\end{array}$ \\
\hline & $n^{*}$ & 275454 & 344141 & 334141 & 323232 & 313232 & 353737 \\
\hline \multirow{4}{*}{$\mathrm{K}=4000$} & $p^{*}$ & 34.8637 .4937 .49 & 34.2836 .6436 .64 & 34.4036 .7736 .77 & & 33.7236 .1336 .13 & 33.8736 .1336 .13 \\
\hline & $\delta^{*}$ & $126.38 \quad 209.88209 .88$ & 130.48211 .00211 .00 & $\begin{array}{lll}129.51 & 210.87 & 210.87\end{array}$ & & 135.03211 .46211 .46 & 133.58211 .61211 .61 \\
\hline & $\pi^{*}$ & $30.25 \quad 126.22 \quad 126.22$ & $31.93 \quad 120.35 \quad 120.35$ & 34.58124 .09124 .09 & & $42.46129 .22 \quad 129.22$ & 40.36129 .50129 .50 \\
\hline & $n^{*}$ & 141818 & 152020 & 141919 & & 141818 & 141818 \\
\hline \multirow{4}{*}{$\mathrm{K}=5600$} & $p^{*}$ & $35.23 \quad 37.5237 .52$ & 35.1338 .1238 .12 & $35.2938 .28 \quad 38.28$ & $34.8937 .72 \quad 37.72$ & 34.8637 .6837 .68 & \\
\hline & $\delta^{*}$ & $122.70 \quad 210.20 \quad 210.20$ & 124.99208 .88208 .88 & $123.71 \quad 208.72 \quad 208.72$ & 126.53209 .45209 .45 & 126.71209 .51209 .51 & \\
\hline & $\pi^{*}$ & 8.5098 .0398 .03 & $\begin{array}{llll}11.56 & 106.74 & 106.74\end{array}$ & $\begin{array}{llll}15.44 & 112.04 & 112.04\end{array}$ & $24.86119 .02 \quad 119.02$ & $\begin{array}{llll}25.10 & 119.12 & 119.12\end{array}$ & \\
\hline & $n^{*}$ & $13 \quad 18 \quad 18$ & 131616 & $12 \quad 15 \quad 15$ & $11 \quad 14 \quad 14$ & $10 \quad 17 \quad 17$ & \\
\hline
\end{tabular}

Table 2: Equilibria under six seasonality patterns and four setup cost values

lower direct price sensitivity $10=\frac{\partial \delta_{i}^{i}(p)}{\partial p^{1}}=b^{1}<b^{2}=b^{3}=12$ not withstanding.

While the differences in the price equilibria are relatively small, they often have very significant impacts on equilibrium volumes and in particular equilibrium profits. For example, when $K_{t}^{i}=$ 4000, firm 1's profit is nearly $25 \%$ larger under the cyclic seasonality pattern (VI) than under constant demands (I). When $K_{t}^{1}=5400$ firm 1 more than doubles its profit when moving from the constant demand pattern (I) to a pattern with a holiday season at the beginning (IV). The profit increases for firm 2 and 3, when moving from (I) to (IV), are in absolute terms, approximately equal to that experienced by firm 1, even though in relative terms they amount to an increase of approximately $16 \%$.

It is hard to predict which seasonality pattern results in higher equilibrium prices: for $K_{t}^{1}=500$, the prices under the constant pattern (I) are lower than those under the cyclic pattern (VI) but the opposite is true for $K_{t}^{i}=4000$. Note, also, that the impact of a fixed order cost increase on the equilibrium prices may vary rather significantly depending on which of the seasonality patterns prevails. For example, when K increases from 500 to 1000, equilibrium prices increase by $\$ 1.26$ for firm 1, but by only $\$ 0.09$ for firms 2 and 3 under (I), while the price increases under (VI) are $\$ 0.14$ for firm 1 and $\$ 0.33$ for firms 2 and 3. The order pattern, in general, and the number of order periods, in particular, vary greatly by firm and by seasonality pattern, see for example the case where $K_{t}^{i}=1000$.

When $K_{i}^{t}=4000$, a unique equilibrium continues to exist under all seasonality patterns except for (IV). Here, there are at least two equilibria $p_{1}^{*}=[34.03 ; 36.28 ; 36.40]$ and $p_{2}^{*}=[34.03 ; 36.40 ; 36.28]$. 


\begin{tabular}{|c|c|c|c|c|c|c|c|}
\hline & & (I) & (II) & (III) & (IV) & $(\mathrm{V})$ & $(\mathrm{VI})$ \\
\hline \multirow{4}{*}{$\mathrm{K}=5000$} & $p^{*}$ & $42.8638 .58 \quad 38.58$ & 39.5039 .8539 .85 & 39.7439 .9739 .97 & 36.8441 .0741 .07 & 39.0041 .0741 .07 & 38.9940 .0740 .07 \\
\hline & $\delta^{*}$ & $27.41 \quad 123.00123 .00$ & $32.34 \quad 120.57 \quad 120.57$ & $32.00120 .57 \quad 120.57$ & $37.26 \quad 118.46 \quad 118.46$ & $33.48 \quad 119.38 \quad 119.38$ & $\begin{array}{llll}33.20 & 120.18 & 120.18\end{array}$ \\
\hline & $\pi^{*}$ & $24.84 \quad 129.65 \quad 129.65$ & $25.78 \quad 136.61 \quad 136.61$ & $26.13 \quad 137.59 \quad 137.59$ & $28.04 \quad 144.57 \quad 144.57$ & $28.11 \quad 145.82 \quad 145.82$ & $\begin{array}{llll}27.78 & 140.46 & 140.46\end{array}$ \\
\hline & $n^{*}$ & 185454 & 224444 & 214343 & 233232 & 193232 & 193737 \\
\hline
\end{tabular}

Table 3: Equilibria under Cobb-Douglas demand functions (Example 4)

(These equilibria are both interior points of the feasible region.) The tatônnement scheme may, in this case, converge to either one of the two equilibria or it cycles through the points $p_{1}^{*}=$ $[34.02 ; 36.40 ; 36.40]$ and $p_{2}^{*}=[34.04 ; 36.28 ; 36.28]$.

When $K_{i}^{t}=5600$, an equilibrium fails to exist for pattern (VI): the tatônnement scheme never converges, irrespective of the starting point. Instead, the scheme cycles between the two (price) points $p_{1}^{*}=[35.24 ; 36.54 ; 36.54]$ and $p_{2}^{*}=[35.25 ; 36.60 ; 36.60]$.

We conclude that the seasonality patterns of the demand functions (as well as the cost parameters) may result in significant differences in the equilibria. Sometimes, the seasonality pattern determines whether a unique equilibrium, no equilibrium or multiple equilibria prevail. The latter two cases arise, of course, in settings where conditions $(\mathrm{C})$ and $(\mathrm{C} 2)$ are violated.

Finally, Table 3 below exhibits the equilibria in Example 4 with $K=5000$. In this case, the seasonality pattern has a significantly larger impact on the equilibrium prices; for example, that of firm 1 decreases by $\$ 6$ when going from pattern (I) to (IV). While firm 1 generally positions itself at a lower price than its competitors, the reverse is true under pattern (I).

\section{$6 \quad$ A generalized model}

In our base model, the time-dependence of the demand functions is expressed by multiplicative seasonality factors only, as in (3), and the fixed costs are constant over time. We have shown that in this base model the optimal sequence of order periods, given a total number of orders is independent of the prices charged by the firms, see (9) and (10).

This decomposability breaks down when the fixed order costs are time-dependent or the demand functions' time-dependence is more general, e.g. when there are additive seasonality terms:

$$
d_{t}^{i}(p)=\alpha_{t}^{i}+\beta_{t}^{i} \delta^{i}(p) \quad t=1, \ldots, T ; \quad t=1, \ldots, N
$$


Under this structure, the best response problem becomes considerably more difficult, albeit that the recent algorithm by VAN DEN Heuvel and Wagelmans (2004) solves it in $O\left(T^{3} \log T\right)$ time. The cost function $C^{i}(p)$ can still be expressed as a piecewise linear, concave function of the deseasonalized demand volume, see the proof of Lemma 1 , but the representation requires $\left(2^{T}-1\right)$ linear prices. This cost function can still be approximated by a power function as in (43) resulting in simple conditions for the existence and uniqueness of equilibria in the approximate oligopoly model - but the tight arguments resulting in this approximation for the basic model no longer apply and the approximation is less accurate.

However, thanks to the availability of the best response algorithm of VAN DEN HEUVEL and WAGELmans (2004), the tatônnement scheme can still be applied effectively to the fully general model. When convergent, its limit point is, of course, a Nash equilibrium. If convergent to the same limit point, regardless of its starting point $p_{(0)}$, the equilibrium is in fact unique. Thus, the tatônnement scheme provides an algorithmic mechanism to establish the existence of a Nash equilibrium in any given instance of the general model. Indeed, we have applied the scheme to a large variety of problem instances of the general model and have found that a unique equilibrium exists in the vast majority of cases.

To illustrate this, we analyze a set of six problem instances with additive rather than multiplicative seasonality factors for the demand function, i.e. $\beta_{t}^{i}=1$. The six instances are obtained from the base case in Example 2 (where all $\alpha_{t}^{i}=0$ ), merely by varying the $\left\{\alpha_{t}^{i}\right\}$ terms. The seasonality patterns are the direct analog of (I)-(VI) in the case of multiplicative seasonalities.

For each pattern, we obtain the $\alpha_{t}^{i}$-terms from the $\beta_{t}^{i}$-factors for the same pattern, employing the transformation $\alpha_{t}^{i}=100\left(\beta_{t}^{i}-1\right)$. (Note that $\frac{1}{54} \sum_{t=1}^{54} \alpha_{t}^{i}=0 \forall i$.) Table 4 displays the equilibrium prices, demand values, profits and number of setups for the 6 instances. As in the case of multiplicative seasonalities, we note that the differences in the equilibrium prices are modest. At the same time, major differences in the number of setup periods may arise, translating into equilibrium profit differences of up to $8.4 \%$. The differences become even larger when the setup cost parameters $K_{t}^{i}$ are increased, see Table 4. As in the case of multiple seasonalities, all firms may be better off under seasonally varying demands or cost parameters, once again contradicting common folklore. 


\begin{tabular}{|c|c|c|c|c|c|c|c|}
\hline & & (I) & (II) & (III) & (IV) & $(\mathrm{V})$ & $(\mathrm{VI})$ \\
\hline \multirow{4}{*}{$\mathrm{K}=1000$} & $p^{*}$ & 32.0533 .0033 .00 & 31.9133 .8733 .87 & 31.9533 .8733 .87 & 32.0434 .7534 .75 & 32.0434 .7534 .75 & $32.0833 .72 \quad 33.72$ \\
\hline & $\delta^{*}$ & $78.57 \quad 116.66 \quad 116.66$ & $80.29115 .65 \quad 115.65$ & $80.04 \quad 115.67 \quad 115.67$ & $80.51 \quad 114.77 \quad 114.77$ & $80.51 \quad 114.77 \quad 114.77$ & $\begin{array}{llll}79.21 & 115.90 & 115.90\end{array}$ \\
\hline & $\pi^{*}$ & $87.33 \quad 156.04 \quad 156.04$ & $88.77 \quad 165.83 \quad 165.83$ & $89.10166 .06 \quad 166.06$ & $\begin{array}{lll}94.63 & 177.07 & 177.07\end{array}$ & $\begin{array}{llll}94.63 & 177.07 & 177.07\end{array}$ & $89.56 \quad 165.68 \quad 165.68$ \\
\hline & $n^{*}$ & 275454 & 324343 & 314343 & 313232 & 313232 & 364545 \\
\hline
\end{tabular}

Table 4: Equilibria under six $\alpha$-patterns

\section{Conclusions}

We have developed the first oligopoly model combining (I) the complexity of time-varying demand and cost functions with (II) that of scale economies due to dynamic lot sizing costs. When the firms engage in price competition, we have identified general sufficient conditions under which a Nash equilibrium exists, under which this Nash equilibrium is unique and under which a simple tatônnement scheme is guaranteed to converge to the equilibrium. In this scheme, firms repeatedly solve their best response problem, i.e. each firm determines the best combined price and dynamic lot sizing policy in response to the assumed price levels of its competitors. The above results were obtained for the case where each firm's demand function in some period $t=1, \ldots, T$ is given by a time-invariant (deseasonalized) demand function, multiplied by a firm dependent seasonality factor for period $t$. In addition, we have assumed that the fixed order costs are constant throughout the planning horizon. (However, in $\S 6$ we have discussed how our results can be extended under more general seasonality patterns and time-dependent fixed costs.)

A single best response problem can be solved with $O\left(T^{2}\right)$ elementary operations and $O(T)$ evaluations of the inverse derivative revenue functions $R_{i}^{\prime-1}(\cdot)$. (For many important classes of demand functions, e.g. the linear and Cobb-Douglas functions, this inverse derivative revenue function can be obtained in closed form). Moreover, only in the first iteration of the tatônnement scheme, do the best response problems require $O\left(T^{2}\right)$ operations. In subsequent iterations, the best response problem is of $O(T)$ complexity.

It is of interest to compare these results with those pertaining to the simpler settings in which only one of the two complicating factors (I) and (II) prevails. The case where all cost parameters and demand functions are time invariant was covered by BERnstein and FEDERGRUEN (2003), albeit in an infinite horizon setting. We have shown that the conditions for existence of an equilibrium or its uniqueness, in our model, are very similar, if not identical, to those required in the special case of time-invariant cost and demand functions. 
In the absence of fixed order costs, the demand in any given period $t$ is optimally procured in a period $s \leq t$ for which the total procurement cost rate $(=$ order cost rate plus holding costs, if any) is lowest, irrespective of the demand values and hence irrespective of the prices charged by the firms. As a consequence, the minimum total procurement cost for firm $i$ is proportional to $\delta^{i}(p)$, its deseasonalized demand volume. Thus, in the absence of fixed order costs, the oligopoly model reduces to the standard price competition model in economics. Since the cost function is linear in $\delta^{i}(p)$, the profit function $\pi^{i}(p)$ is concave in $p^{i}$ if and only if the gross revenue function is concave in $p^{i}$; see Milgrom and Roberts (1990) for a list of various classes of demand functions for which this is the case, so that a Nash equilibrium exists, see (C).

Our model assumes that prices remain constant throughout the planning horizon and we have argued in $\S 1$ why this assumption is often satisfied. The above not withstanding, we hope that our work will be extended to allow for a limited or an arbitrary number of price changes.

\section{References}

[1] Aggarwal, A., J.K. Park. 1993. Improved algorithms for economic lot size problems. Operations Research 41 549-571.

[2] Bernstein, F., A. Federgruen. (2003). Pricing and Replenishment Strategies in a Distribution System with competing Retailers. Operations Research 51 409-426.

[3] Bernstein, F., A. Federgruen. (2004). A General Equilibrium Model for Industries with Price and Service Competition. Operations Research 52 868-886.

[4] Blinder, A.S., E.R.D. Canetti, D.E. Lebow, J.B. Rudd. 1998. Asking About Prices, Russell Sage Foundation, New York.

[5] Cachon, G., P. Harker. 2002. Competition and outsourcing with scale economies. Management Science 48 1314-1333.

[6] Cachon, G. 2003. Supply Chain Coordination with Contracts. In Graves, S.C., A.G. DE KoK: Handbook of Operations Management, Elsevier Publishing Company, Amsterdam, Netherlands. 
[7] Carlton, D.W. 1986. The Rigidity of Prices. American Economic Review 76 637-658.

[8] Cecchetti, S. G. 1986. The Frequency of Price Adjustment: A Study of the Newsstand Prices of Magazines, Journal of Econometrics 31 255-274.

[9] Chevalier, J.A., A.K. Kashyap, P.E. Rossi. 2003. Why Don’t Prices Rise during Periods of Peak Demand? Evidence from Scanner Data. American Economic Review 93 15-37.

[10] Clarke, D.G., R. J. Dolan. 1984. A Simulation Analysis of Alternative Pricing Strategies for Dynamic Environments; I. Introduction. The Journal of Business 57 1792-201.

[11] Dun and Bradstreet. 2000-2001. Industry Norms and Key Business Ratios. Dun and Bradstreet Credit Services, New York.

[12] Dockner, E., S. Jorgensen. 1988. Optimal Pricing Strategies for New Products in Dynamic Oligopolies. Marketing Science 7 315-334.

[13] Eliashberg, J., R. Steinberg. 1993. Marketing-production joint decision-making. In EliashberG, J., Lilien, G.L. (eds.): Handbooks in Operations Research and Management Science: Vol.5: Marketing. Amsterdam: North-Holland, 827-880.

[14] Eliashberg, J., A.P. Jeuland. 1986. The Impact of Competitive Entry in a Developing Market upon Dynamic Pricing Strategies. Marketing Science 5 20-36.

[15] Elmaghraby, W., P. Keskinocak. 2003. Dynamic Pricing in the Presence of Inventory Considerations: Research Overview, Current Practices and Future Directions, Management Science 49 1287-1309.

[16] Federgruen, A., M. Tzur. 1991. A Simple Forward Algorithm to solve General Dynamic Lot-Sizing Models with n Periods in O(n log n) or O(n) time. Management Science 37 909-925.

[17] Gilbert, S.M. 1999. Coordination of Pricing and Production Planning for Constant Priced Goods. European Journal of Operational Research 114 330-337.

[18] Kachani, S., G. Perakis, C. Simon. 2004. An MPEC Approach to Dynamic Pricing and Demand Learning. Working Paper, MIT, Cambridge, MA. 
[19] Kalish, S. 1983. Monopolist Pricing with Dynamic Demand and Production Cost. Marketing Science 2 135-160.

[20] Kashyap, A.K. 1995. Sticky Prices: New Evidence from Retail Catalogs. Quarterly Journal of Economics $110245-274$.

[21] Kunreuther, H., L. Schrage. 1973. Joint Pricing and Inventory Decisions for Constant Priced Items. Management Science 19 732-738.

[22] Milgrom, P., J. Roberts. 1990. Rationalizability, Learning, and Equilibrium in Games with Strategic Complementarities. Econometrica 58 1255-1277.

[23] Perakis G., A. Sood. 2003. Competitive Multi-Period Pricing with Fixed Inventories. Working Paper, MIT, Cambridge, MA.

[24] Perakis G., A. Sood. 2004. Multiperiod Pricing for Perishable Products: A Robust Optimization Approach. Working Paper, MIT, Cambridge, MA.

[25] RAO, R.C., F.M. BAss. 1985. Competition, strategy, and price dynamics: A theoretical and empirical investigation. Journal of Marketing Research 22 283-296.

[26] Tellis, G.J. 1988. The price sensitivity of competitive demand: A meta analysis of sales response models. Journal of Marketing Research 15 331-341.

[27] Thomas, J. 1970. Price production decisions with deterministic demand. Management Science $16747-750$.

[28] Tirole, J. 1988. The Theory of Industrial Organization. MIT Press, Cambridge, MA.

[29] Van Hoesel, C.P.M., A.P.M. Wagelmans. 1992. Economic Lot Sizing: An (n $\log n$ ) Algorithm that runs in linear Time in the Wagner-Whitin Case. Operations Research $40145-$ 156.

[30] Van den Heuvel, W., A.P.M. Wagelmans. 2004. A polynomial time algorithm for a deterministic joint pricing and inventory model. Working Paper, Erasmus University, Rotterdam, Netherlands. 
[31] Vives, X. 2000. Oligopoly Pricing. MIT Press, Cambridge, MA.

[32] Wagner, H.M. 1960. A postscript to 'dynamic problems of the firm.' Naval Research Logistics Quarterly 7 7-12.

[33] Wagner, H.M., T.M. Whitin. 1958. Dynamic Version of the Economic Lot Size Model. Management Science 5 89-96.

[34] Wagner, H.M., T.M. Whitin. 1959. Comments on 'Economic Lot Sizes with Seasonal Demand.' Operations Research 7394. 